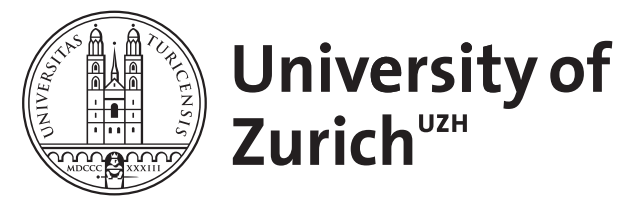

\title{
Therapie der Belastungsinkontinenz
}

\author{
Hanzal, E ; Scheiner, D
}

\begin{abstract}
Fallvignette: Die 35-jährige Buschauffeurin Petra K., Para 2, berichtet über Harnverlust beim Husten, Niesen, Lachen und schwerem Heben. Sie geht achtmal täglich auf die Toilette und muss in der Nacht nicht aufstehen. Sie hat wegen der Inkontinenz mit ihren Turnstunden aufgehört. Sie ist $162 \mathrm{~cm}$ gross und wiegt $83 \mathrm{~kg}$ (BMI 32,4). Im Rahmen einer Erstuntersuchung bei ihrer Frauenärztin wurde die Diagnose einer Belastungsinkontinenz gestellt. Bisher wurde noch keine Therapie durchgeführt. Grundlegende Prinzipien: Die Therapieplanung bei Belastungsinkontinenz hängt von der Anamnese, dem Leidensdruck und den Wünschen der Betroffenen, der Erfahrung der Behandler und dem aktuellen Wissensstand ab. Merke: Grundsätzlich gilt bei der Therapie der Belastungsinkontinenz das Prinzip: konservativ vor operativ.
\end{abstract}

Posted at the Zurich Open Repository and Archive, University of Zurich

ZORA URL: https://doi.org/10.5167/uzh-46189

Book Section

Accepted Version

Originally published at:

Hanzal, E; Scheiner, D (2010). Therapie der Belastungsinkontinenz. In: Tunn, R; Hanzal, E; Perucchini, D. Urogynäkologie in Praxis und Klinik (2. Aufl.). Berlin, DE: de Gruyter, 161-184. 


\title{
16 Therapie der Belastungsinkontinenz
}

\author{
Engelbert Hanzal, David Scheiner, K. Lobodasch, Daniele Perucchini, K. Tamussino
}

\begin{tabular}{|c|l|}
\hline 25 v. Chr. & Pessar aus Bronze (Celsus) \\
\hline 1900 & Harnröhrenunterspritzung mit Paraffin \\
\hline 1907 & Grazilisplastik (Giordano) \\
\hline 1913 & Paraurethralnähte (Kelly) \\
\hline 1948 & Beckenbodentraining (Kegel) \\
\hline 1949 & abdominelle Kolposuspension (Marshall, Marchetti, Krantz) \\
\hline 1959 & Nadelsuspension (Peyrera) \\
\hline 1961 & Modifikation der Kolposuspension (Burch) \\
\hline 1995 & Tension-free vaginal tape (Petros, Ulmsten) \\
\hline 2001 & Transobturatorischer Zugang (Delorme) \\
\hline 2002 & Duloxetin \\
\hline 2006 & kurze synthetische Schlingen \\
\hline
\end{tabular}

\section{Fallvignette}

Die 35-jährige Buschauffeurin Petra K., Para 2, berichtet über Harnverlust beim Husten, Niesen, Lachen und schwerem Heben. Sie geht achtmal täglich auf die Toilette und muss in der Nacht nicht aufstehen. Sie hat wegen der Inkontinenz mit ihren Turnstunden aufgehört. Sie ist $162 \mathrm{~cm}$ groß und wiegt $83 \mathrm{~kg}$ (BMI 32,4). Im Rahmen einer Erstuntersuchung bei ihrer Frauenärztin wurde die Diagnose einer Belastungsinkontinenz gestellt. Bisher wurde noch keine Therapie durchgeführt.

\subsection{Grundlegende Prinzipien}

Die Therapieplanung bei Belastungsinkontinenz hängt von der Anamnese, dem Leidensdruck und den Wünschen der Betroffenen, der Erfahrung der Behandler und dem aktuellen Wissensstand ab.

Merke: Grundsätzlich gilt bei der Therapie der Belastungsinkontinenz das Prinzip: konservativ vor operativ.

\subsubsection{Konservativ}

Konservative Maßnahmen bestehen aus Änderungen des Lebensstils (sogenannte "Lifestyle-Interventionen" - z. B. Gewichtsreduktion, Änderung des Trinkverhaltens, Nikotinkarenz) und den physiotherapeutischen Maßnahmen (z. B. Beckenbodentraining, Elektrotherapie). Diese Maßnahmen sind am effektivsten, wenn sie von geschulten TherapeutInnen und motivierten Patientinnen durchgeführt werden.

\subsubsection{Medikamentös}

Medikamentöse Behandlungsformen spielten bei der Belastungsinkontinenz traditionell eine untergeordnete Rolle; dies hat sich aber seit der Einführung des Serotonin- und Noradrenalin-Wiederaufnahmehemmers Duloxetin etwas geändert.

\subsubsection{Operativ}

Inkontinenzoperationen sind Paradebeispiel für elektive Eingriffe. Die geringe Morbidität konser- 
vativer Therapieformen sowie die durchaus substanzielle Wahrscheinlichkeit unerwünschter Ergebnisse operativer Verfahren (persistierende Inkontinenz, Rezidiv, Komplikationen) werden daher nur in Ausnahmefällen dazu führen, dass sich Patientin und Behandler für ein primär operatives Verfahren entscheiden.

\subsubsection{Algorithmus}

Das Vorgehen bei Patientinnen, die unter Symptomen einer Belastungsinkontinenz leiden, kann vereinfacht in einem Algorithmus dargestellt werden ( Abb. 16.1).

\subsection{Konservative Therapie}

\subsection{1 Änderung des Lebensstils}

Übergewicht ist ein unabhängiger Risikofaktor für Belastungsinkontinenz, und Gewichtsreduktion ist eine akzeptable Option für übergewichtige Frauen mit diesem Problem (ICl 2005). In einer randomisierten Studie wurden bei Patientinnen mit einem sechsmonatigen Gewichtsreduktionsprogramm die Inkontinenzepisoden um $47 \%$ reduziert (Subak et al. 2009). Weitere Interventionen wie Nikotinkarenz und Koffeinreduktion werden oft empfohlen, haben aber keinen erwiesenen Effekt auf die Belastungsinkontinenz. Maßnahmen zur Änderung

\section{Belastungsinkontinenz}

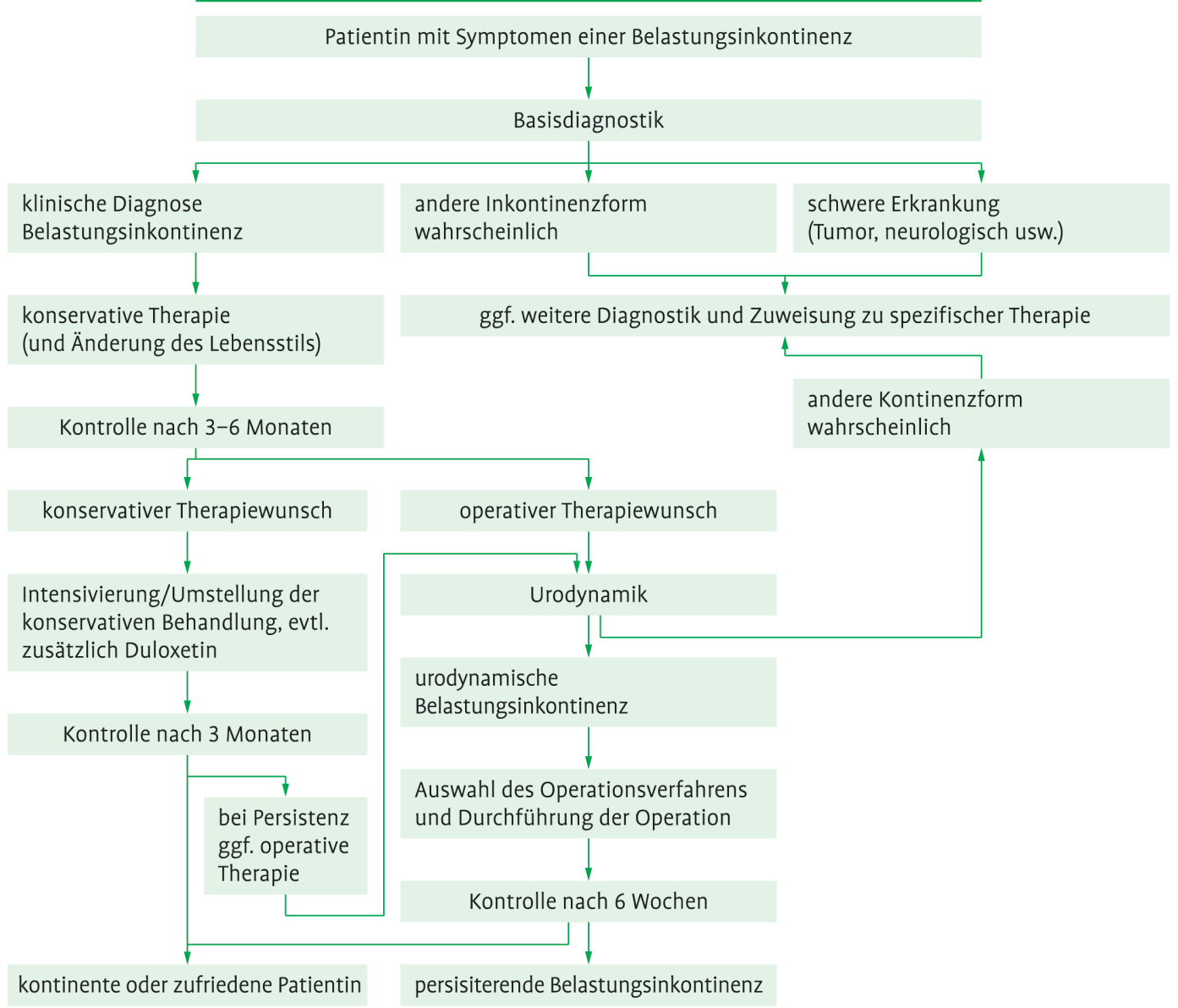

Abb. 16.1 Algorithmus zum Vorgehen bei Patientinnen mit Symptomen einer Belastungsinkontinenz 
des Lebensstils können folgendermaßen gegliedert werden:

\section{Beratung}

Die Beratung und Aufklärung der Patientinnen über die Funktion des Harntraktes und des Beckenbodens sollte auch Faktoren einschließen, die eine Belastungsinkontinenz begünstigen. Dazu gehören Übergewicht, chronische Obstipation, chronischer Husten, Rauchen, Harnwegsinfekte und Medikamente, die aufgrund ihrer Nebenwirkungen mit Belastungsinkontinenz assoziiert sind oder eine Dranginkontinenz zusätzlich fördern können.

\section{Verhaltensschulung, Atemtechnik}

Bei den Aktivitäten des täglichen Lebens wie zum Beispiel Heben und Tragen schwerer Lasten soll die Patientin eine korrekte Haltungs- und Atemtechnik erlernen. Dies wird häufig kombiniert mit einem Haltungs- und Gangtraining. Die Stuhlregulierung kann sich ebenfalls positiv auf die Inkontinenz auswirken. Die Patientinnen müssen über die Notwendigkeit der Langzeittherapie informiert sein. Die Koordination des Diaphragma pelvis mit dem Diaphragma pulmonale kann ebenfalls in eine physiotherapeutischen Behandlung einbezogen werden. Die Patientin lernt, den Beckenboden beim Ausatmen anzuspannen und beim Einatmen zu entspannen. Der Harndrang kann "veratmet" werden und der Gang zur Toilette wird hinausgezögert. Dadurch kann ein koordiniertes Miteinander beider Diaphragmen erleichtert werden.

\subsubsection{Beckenbodentraining und Trainings- hilfen}

Beckenbodentraining ( $\triangleright$ Kap. 15.1) besteht aus wiederholter selektiver willkürlicher Anspannung und Entspannung spezifischer Beckenbodenmuskeln. Dies setzt eine sichere Muskelbeherrschung voraus, sodass die richtigen Muskeln aktiviert und unbeabsichtigte Kontraktionen benachbarter Muskelgruppen vermieden werden.

Die Harn- und die Stuhlinkontinenz können durch ein Beckenbodentraining gebessert, manchmal auch geheilt werden. Viele Therapieprogramme folgen einem 3-Stufen-Konzept:

1. Übungen zur Wahrnehmung der Beckenbodenmuskulatur
2. Integration der Muskulatur in ein ganzheitliches Körperkonzept, reflex- und situationsgerechtes Aktivieren und Entspannen der Muskeln

3. Kräftigung der Beckenbodenmuskulatur und Einüben der Reflexe durch Integration in den Alltag (Langzeitprogramm)

Eine alleinige Verordnung des Arztes und die Mitgabe von Informationsblättern mit Übungsanleitungen erfüllen diese Voraussetzungen nur unzureichend und sind damit auch nicht so erfolgreich wie ein Beckenbodentraining mit entsprechender fachlicher Anleitung.

Es hat sich als praktikabel erwiesen, die Patientinnen hinsichtlich der Reaktionsfähigkeit des Beckenbodens in zwei Gruppen aufzuteilen: Frauen mit guter und mit fehlender Kontraktionsfähigkeit. Dies kann durch eine gynäkologische Untersuchung festgestellt werden, während der man die Frau Kneifübungen durchführen lässt. Frauen mit guter Beckenbodenreaktion können sofort mit dem Training beginnen. Bei fehlender Reaktion kann zunächst mit einer Elektrostimulation der Muskulatur begonnen werden. Wird dadurch der Beckenboden reaktiviert, folgt das Trainingsprogramm.

Die Muskelfunktionsprüfung nach dem PERFECT-Schema verdeutlicht auf einen Blick, wie die physiotherapeutischen Behandlungsziele eingesetzt werden können $(\triangleright$ Tab. 16.1). Die Kraft der

Tab. 16.1 Mnemotechnisches Schema zur Strukturierung des Beckenbodentrainings (PERFECT-Schema; Laycock 2002)

\begin{tabular}{|c|c|c|}
\hline $\mathbf{P}$ & Power & $\begin{array}{l}\text { Kraft der Beckenbodenmusku- } \\
\text { latur - Beurteilung von 0-5 } \\
\text { (s. Oxford Grading, Tab. 16.2) }\end{array}$ \\
\hline $\mathbf{E}$ & Endurance & $\begin{array}{l}\text { Ausdauerkraft ca. } 10 \mathrm{~s} \text { halten } \\
\text { (slowtwitch fibres) mit ansch- } \\
\text { ließender Entspannung }\end{array}$ \\
\hline $\mathbf{R}$ & Repetitions & $\begin{array}{l}\text { Wiederholungen der Übung } \\
(10 \text { s) mit anschl. Entspan- } \\
\text { nung, } 3 \times 10 \text { Kontraktionen } \\
\text { pro Tag }\end{array}$ \\
\hline $\mathbf{F}$ & Fast Contractions & $\begin{array}{l}\text { Schnellkraft (fast twitchfibres) } \\
\text { 5-10 schnelle Anspannungen }\end{array}$ \\
\hline $\mathbf{E}$ & Elevation & $\begin{array}{l}\text { Blasenhalselevation: Anheben } \\
\text { der Blase durch Anspannung } \\
\text { des M. levator ani }\end{array}$ \\
\hline C & Cough response & $\begin{array}{l}\text { reflektorische Kontraktion der } \\
\text { Beckenbodenmuskulatur beim } \\
\text { Husten }\end{array}$ \\
\hline $\mathbf{T}$ & Transcribe it all & Dokumentation aller Ergebnisse \\
\hline
\end{tabular}


Tab. 16.2 Oxford-Schema zur digitalen transvaginalen Beurteilung der Beckenbodenkontraktilität (Sampselle et al. 1989)

\begin{tabular}{ll}
\hline Grad & Kraft \\
\hline 0 & keine \\
1 & angedeutet \\
2 & schwach \\
3 & mäßig \\
4 & gut \\
5 & sehr gut \\
\hline
\end{tabular}

Beckenbodenmuskulatur wird nach dem OxfordSchema beurteilt $(\downarrow$ Tab. 16.2$)$.

Nach dem Erlernen der Muskelbeherrschung erfolgt das Muskeltraining.

Merke: Dauer der Kontraktion, Dauer der Intervalle, Anzahl der Kontraktionen pro Übungen, Anzahl der Kontraktionen pro Tag sowie die Gesamttrainingsdauer sollten der Patientin als individueller Trainingsplan vorgegeben werden.

Als allgemeine Empfehlung mögen drei Trainingseinheiten pro Tag mit jeweils zehn Kontraktionen gelten. Dabei sollten die maximalen Kontraktionen über sechs Sekunden gehalten werden und eine mindestens sechs Sekunden währende Pause folgen. Diese täglichen Übungen sollten über sechs Monate in dieser Intensität fortgesetzt werden. Das Beckenbodentraining zu Hause soll einmal pro Woche durch eine Beckenbodentraining unter physiotherapeutischer Kontrolle und Anleitung ergänzt werden. Eine Trainingseinheit umfasst üblicherweise 45 Minuten, wobei neben den maximalen langen Kontraktionen über sechs Sekunden auch häufige schnelle Kontraktionen geübt werden sollten.

Beim Biofeedback $($ Kap. 15.2) erhalten Patientin und Therapeuten durch visuelle, auditive oder taktile Signale Rückmeldungen über normalerweise unbewusst ablaufende physiologische Prozesse. Das Signal wird dann in einem Erziehungsprozess genutzt, um ein spezifisches therapeutisches Ziel zu erreichen. Das Signal wird quantitativ dargestellt und der Patient angeleitet, die physiologischen Abläufe zu beeinflussen und damit zu kontrollieren. Als Technik steht die Registrierung von Druck und EMG als physiologische Parameter zur Verfügung.
Auch hier sind Instruktionen, wie das Signal beeinflusst werden kann, von großer Bedeutung. Ziel des Biofeedbacks ist, die spezifische Dysfunktion des unteren Harntraktes, in der Regel die Hyporeaktivität der Sphinkter-/Beckenbodenmuskulatur, durch zunehmende Bewusstmachung zu verbessern. Bei der Belastungsharninkontinenz bedeutet dies, auf direktem Wege die reflektorische Kontraktionsleistung über Training der Kontraktionsdauer und -kraft zu verbessern und zu erlernen, um im richtigen Moment reagieren zu können. Beckenbodentraining mit Biofeedback als Kombinationstherapie hat sich gegenüber dem Beckenbodentraining alleine in den bisher durchgeführten Untersuchungen jedoch nicht als überlegen erwiesen.

Für die allgemeine Konditionierung und Gewichtsreduktion sind Ausdauersportarten wie Schwimmen, Radfahren oder Nordic Walking zu empfehlen.

Die Anwendung von Vaginalkonen $($ Kap. 15.5) bildet ein ähnliches Verfahren wie das Beckenbodentraining mit Biofeedback. Zu einem Set gehören gleich große Konen mit unterschiedlichen Gewichten zwischen 20 und 100 g. Diese Methode bewährt sich vor allem in Situationen, in denen ein Zugang zu fachgerechter physiotherapeutischer Anleitung nicht gegeben ist.

\subsubsection{Elektrostimulation}

Bei der Elektrotherapie ( $\$$ Kap. 15.3) werden über Elektroden elektrische Impulse übertragen. Man geht davon aus, dass die Wirkung umso effektiver ist, je näher die Elektroden am Nervus pudendus liegen, der die entscheidenden Organe mitversorgt. Das wird am effizientesten über Oberflächenelektroden erreicht, die kutan, vaginal, rektal oder urethral eingesetzt werden können. Häufig wird die Elektrostimulation auch als Initialtherapie beim Beckenbodentraining eingesetzt. Angewandt werden kann die Elektrotherapie sowohl in Kombination mit anderen Therapieformen als auch allein.

Folgende Anwendungsarten sind gebräuchlich:

- niederfrequenter Strom:

- $50 \mathrm{~Hz}$ Langzeitstimulation; Anwendung bei Belastungsinkontinenz

- 10-20/50 Hz Stimulation; Anwendung bei Belastungs- und Drang-Harninkontinenz

- mittelfrequenter Strom:

- Interferenzstrom; Anwendung bei Stressund/oder Drang-Harninkontinenz (vor allem im höheren Lebensalter) 
- hochfrequenter Strom:

- Kurzwelle, Mikrowelle; Anwendung zur Verbesserung der Durchblutung, allgemeinen Entspannung und Entkrampfung im DetrusorSphinkter-Bereich

Durch die Stimulation wird die quer gestreifte Muskulatur des Beckenbodens abwechselnd kontrahiert und relaxiert. Es erfolgt somit ein passives Beckenbodentraining. Bei der Belastungsinkontinenz werden Frequenzen von $50 \mathrm{~Hz}$ und mehr angewendet. Moderne Geräte sind batteriebetrieben und für die Heimnutzung geeignet. Die Patientin kann Impuls- und Stromstärke selbst regulieren. Eine genaue Einweisung ist jedoch erforderlich.

Folgende Kontraindikationen müssen beachtet werden:

- Menstruation und Zwischenblutungen

- Schwangerschaft, Harnverhalt

- Harnwegsinfektionen

- Urogenitalfistel

- schwere Herzrhythmusstörungen

- Metallimplantate im Anwendungsgebiet

- Herzschrittmacher

\subsubsection{Pessartherapie}

Bei Harninkontinenz können spezielle Ringpessare angewendet werden, die mit einer Verdickung den urethrovesikalen Übergang anheben sollen $(\triangleright$ Abb. 16.2). Außerdem kommen Einmalmodelle verschiedenster Bauart und aus diversesten Materialien zur Anwendung ( Kap. 20.1) (Stöpsel für die Harnröhre sind aufgrund erhöhter Infektionsraten obsolet.).

Merke: Ein Kriterium in der Pessartherapie ist eine leichte Handhabung durch die betroffene Frau selbst. Dies führt zu einer verbesserten Akzeptanz und reduziert das Risiko von Infektionen und Druckulzerationen.

Voraussetzung dafür ist, dass wieder verwendbare Pessare regelmäßig entfernt und gepflegt und Einmalpessare wie vorgesehen ausgetauscht werden. Unter optimalen Voraussetzungen profitieren junge Frauen ebenfalls von der Pessartherapie. Auch im hohem Alter, bei Inoperabilität, schlechtem Allgemeinzustand oder auf Wunsch der Patientin kann die Pessartherapie gute Dienst leisten.

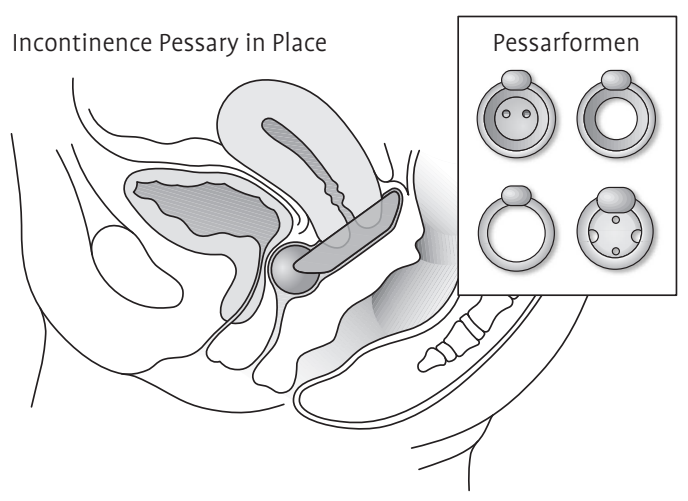

Abb. 16.2 Inkontinenzpessare verschiedener Bauart (Bild: Rogers 2008)

Voraussetzung ist eine ausreichende Motivation und Compliance der Patientin. Auch ältere Frauen sollten den selbstständigen Umgang mit dem Pessar erlernen. Die Anpassung erfolgt individuell durch den behandelnden Arzt. An Nebenwirkungen sind lokale Ulzerationen, Blutungen und Harnwegsinfektionen zu berücksichtigen. Zur besseren Handhabbarkeit sind Gleitmittel hilfreich, oft werden östrogenhaltige Cremes empfohlen. Inkontinenztampons aus Schaumstoff können zur Überbrückung besonderer Belastungssituationen oder längerer Abwesenheit aus dem häuslichen Milieu eingesetzt werden.

\subsection{Medikamentöse Therapie}

\subsection{1 Östrogene}

Die Hormonersatztherapie in der Behandlung der Belastungsinkontinenz hat an Bedeutung verloren.

Merke: Seit der Publikation der HERS-Studie, die eine Verschlechterung von Inkontinenzsymptomen unter oraler Hormontherapie zeigte, kann diese Behandlungsform bei der Belastungsinkontinenz nicht mehr empfohlen werden (Grady et al. 2001).

Die Situation bei lokaler Gabe von Östriol ist weniger klar, wenige Experten würden diese Therapieform jedoch als alleinige oder primäre Behandlung der Belastungsharninkontinenz empfehlen. 


\subsubsection{Duloxetin}

Der Serotonin- und Noradrenalin-Wiederaufnahmehemmer Duloxetin ist seit August 2004 für die medikamentöse Therapie in den EU-Ländern zugelassen (der Zulassungsantrag in den USA wurde zurückgezogen). Duloxetin erhöht die Aktivität des Nervus pudendus im Bereich des Onuf-Kernes im Sakralmark. In Kombination mit einem Beckenbodentraining zeigte sich unter Studienbedingungen eine Verbesserung der Lebensqualität belastungsinkontinenter Frauen. Auf die Nebenwirkungen, die überwiegend serotoninerger Natur sind, sind die Patientinnen vorher hinzuweisen, besonders auf die initial auftretende passagere Übelkeit.

Duloxetin gibt es als 20- und 40-mg-Kapseln. Die einschleichende Dosierung wird empfohlen, um Nebenwirkungen wie Übelkeit abzuschwächen.

Die Ansprechrate ist bei Patientinnen jenseits des 65. Lebensjahres geringer. Komorbiditäten und Komedikationen erfordern vor der Verordnung von Duloxetin eine gründliche Anamneseerhebung und eine individuelle Indikationsstellung bzw. Dosierung. Andererseits besteht im Alter häufig eine geringere Compliance für andere Therapieformen wie Physiotherapie oder Pessartherapie. Die International Consultation on Incontinence (ICl) hat Duloxetin in ihre Behandlungsguidelines für Harninkontinenz aufgenommen.

In der Alltagssituation weichen sowohl Erfolgsraten als auch Nebenwirkungsraten erheblich von denen unter Studienbedingungen ab. So konnte bei 222 Frauen in einer Anwendungsbeobachtung nur bei $37 \%$ eine Verbesserung erzielt werden, während Nebenwirkungen bei $71 \%$ auftraten und $66 \%$ die Therapie abbrachen (Duckett et al. 2007).

\subsubsection{Alphaadrenorezeptor-Agonisten}

Alphaadrenorezeptoren finden sich in der glatten Muskulatur der Urethra und des Blasenhalses. Eine Behandlung mit diesen Substanzen erhöht den urethralen Verschlussdruck, verursacht aber kardiovaskuläre Nebenwirkungen, Hypertonie, Tachykardie und Arrhythmie. Zusätzlich können Schlafstörungen, Kopfschmerzen und Tremor auftreten. Langzeitdaten dieser Therapie fehlen.

\subsection{Operative Therapie}

\subsubsection{Historischer Abriss}

Die operative Ära der Belastungsinkontinenz begann vor etwa einem Jahrhundert. Kelly schlug
1913 vor, das geschwächte Gewebe beim Blasenhals zu raffen (Kelly plication), um ein suburethrales Widerlager zu schaffen (Kelly 1913). Seine Methode erzielt jedoch Langzeitresultate von lediglich $60 \%$ (Bergman et al. 1995). Zeitgleich wurden in Europa die abdominalen Schlingentechniken entwickelt (Frangenheim et al. 1914, Goebell et al. 1910, Stoeckel et al. 1917). 1907 beschrieb von Giordano die Urethralschlinge mittels des M. gracilis. Die Grundlage der heutigen pubovaginalen Schlingentechnik hat Aldridge 1942 beschrieben (Aldridge 1942): Ein Streifen Rektusfaszie wird unterhalb der Harnröhre befestigt, um einen Widerstand in Situationen mit erhöhtem Abdominaldruck zu liefern.

Der nächste Meilenstein in der Inkontinenzchirurgie bildet die Vesicourethropexie nach MarshallMarchetti-Krantz (MMK) (Marshall et al. 1949). Hierbei wird die endopelvine Faszie und Scheide in eine höhere Position auf Höhe des Blasenhalses oder vesikourethralen Übergangs zur Symphyse resp. den unteren Schambeinästen hin gehoben und am Periost fixiert.

Pereyra beschrieb 1959 erstmals die Blasenhalssuspension mittels einer langen Nadel von vaginal: die vaginale Nadelsuspension (Pereyra 1959), die später von Raz (Raz 1981) und Stamey (Stamey 1973) u. a. m. modifiziert wurde. Im Prinzip wird von vaginal ein nicht resorbierbares Nahtmaterial zur Bauchdecke gezogen und paraurethral beidseits sowie in der Bauchdecke an der endopelvinen Faszie verankert. Die Rezidivrate liegt bei über $40 \%$, sodass diese Operationen heute obsolet sind.

Die im Jahre 1961 eingeführte abdominale Kolposuspension nach Burch (Burch 1961), bei der die Scheide von abdominal her mit Suspensionsfäden nicht mehr ans Periost, sondern seitlich an das Cooper-Ligament (Ligamenta pectinealia) fixiert wird, um die urethrale Hypermobilität zu verhindern, wurde in der Folge Standard. Die Erfolgsquote liegt bei bis $90 \%$ nach einem Jahr und Metaanalysen zeigen Erfolgsraten von $78 \%$ nach 20 Jahren. So weist derzeit in Langzeitbeobachtungen die offene Kolposuspension die höchste Effizienz in der Primär- und Rezidivsituation auf. Unter dem Begriff der abdominalen Kolposuspension sind zahlreiche Modifikationen subsumiert, darunter die häufigste nach Tanagho oder Cowan.

McGuire und Lytton beschrieben 1978 die Einlage eines freien Faszienstückes des $\mathrm{M}$. rectus abdominis suburethral von vaginal als Schlinge im 
Bereich des Blasenhalses. Die abdominale Gewinnung dieses autologen Grafts erhöht aber die Morbidität, sodass nach Allo- oder Xenografts wie Kadaverfaszien (lyophilisierte Durabänder) und zunehmend nach einer idealen künstlichen Schlinge gesucht wurde. Doch solche alloplastische Implantate führten immer wieder zu Erosionen, Invasion in Urethra oder Harnblase oder zu Infektionen, welche die Explantation des Implantates erforderten.

Ulmsten und Petros haben 1995 das tensionfree vaginal tape (TVT) als neue Schlingenoperation eingeführt (Ulmsten und Petros 1995) ( Tab. 16.3). Nicht mehr in Narkose, sondern in Lokalanästhesie und Analgosedation wird ein Kunststoffband über eine kleine suburethrale Kolpotomie midurethral mit einer Spezialnadel retropubisch hochgeführt und über zwei suprasymphysäre Stichinzisionen ausgeleitet. Im Unterschied zur Kolposuspension soll das TVT eine Stabilisierung der mittleren Urethra und nicht eine Reposition des Blasenhalses bewirken. Mehrere randomisiert kontrollierte Studien verglichen das TVT mit dem bisherigem Standard, der abdominalen Kolposuspension. So finden sich für das TVT und die Kolposuspension nach Burch vergleichbare objektive Kontinenzraten von $81 \%$ vs. $90 \%$ nach fünf Jahren (Ward et al. 2008), 11-Jahresresultate mit subjektiven und objektiven Kontinenzraten für das TVT von 90 bis $95 \%$ resp. $77 \%$ (Nilsson et al. 2008) und eine bessere Kosteneffektivität bei kürzerer Operations- und Hospitalisationszeit sowie geringeren Langzeitkomplikationen (Cody et al. 2003, Dean et al. 2006, Kilonzo et al. 2004).

Delorme beschrieb 2001 zur Vermeidung von Komplikationen mit retropubischen Verfahren den ersten transobturatorischen Zugang (Delorme 2001). Inzwischen wurde eine Reihe transobturatorischer Systeme entwickelt, bei denen das jeweilige Band entweder von außen nach vaginal ("outside in") oder von vaginal nach außen (,inside out") gestochen wird.

Tab. 16.3 Entwicklung der Schlingentechniken. Sechs Jahre, nachdem Ulmsten und Petros das TVT aufgrund der Integraltheorie entwickelten, kam 2001 die erste Modifikation auf den Markt, das SPARC. Hierbei sollte die Komplikationsrate dadurch gesenkt werden, dass die Bandeinlage von abdominal nach vaginal erfolgt. Tatsächlich finden sich keine relevanten Unterschiede. Die im selben Jahr vorgestellte präpubische Schlinge hat sich nicht breit durchsetzen können. Das vom Prinzip her interessante adjustierbare Schlingensystem Remeex hat sich, da sehr teuer, ebenfalls nicht durchsetzen können. Auch nach mehreren Jahren kann noch nachjustiert werden. Zu bedenken ist, dass das Adjustiersystem, welches auf der Bauchdeckenfaszie zu liegen kommt, dort in einem sehr beweglichen System ist, was die korrekte Anspannung/Zugeinstellung erschwert. Erst die beiden 2001 resp. 2003 eingeführten transobturatorischen Schlingen brachten eine Neuerung und konnten sich rasch - sogar noch vor Veröffentlichung der Datenlage mittels randomisierter Studien - verbreiten. Ob die neueste Entwicklung der Minischlingen Vorteile birgt, bleibt abzuwarten.

\begin{tabular}{|c|c|c|c|c|c|c|c|}
\hline Jahr & Entwickler & Autor & Band & Prinzip & Basis & Rationale & Verkauf \\
\hline 1995 & Gynäkologie & $\begin{array}{l}\text { Ulmsten } \\
\text { Petros }\end{array}$ & TVT & $\begin{array}{l}\text { Midurethra } \\
\text { retropubisch }\end{array}$ & Integraltheorie & $\begin{array}{l}\text { Dysbalance im } \\
\text { im kleinen Becken } \\
\text { nach Kolposuspension } \\
\text { nach Burch }\end{array}$ & > 2 Mio. \\
\hline 2001 & Urologie & $\begin{array}{l}\text { FDA } 2001 \\
\text { Pub } 2002\end{array}$ & SPARC & $\begin{array}{l}\text { von oben } \\
\text { nach unten }\end{array}$ & Integraltheorie & $\begin{array}{l}\text { weniger } \\
\text { Komplikationen }\end{array}$ & \\
\hline 2001 & Gynäkologie & $\begin{array}{l}\text { Daher } \\
\text { Ulmsten }\end{array}$ & TVT & präpubisch & & Blasen-Perf. & $\begin{array}{l}\text { nicht } \\
\text { etabliert }\end{array}$ \\
\hline 2001 & $\begin{array}{l}\text { Gynäkologie } \\
\text { Urologie }\end{array}$ & Pub 2003 & Remeex & $\begin{array}{l}\text { adjustable } \\
\text { Blasenhals }\end{array}$ & & komplexe Situation & $\begin{array}{l}\text { nicht kassen- } \\
\text { pflichtig }\end{array}$ \\
\hline 2001 & Urologie & Delorme & TOT & $\begin{array}{l}\text { outside-in } \\
\text { Transobturator }\end{array}$ & $\begin{array}{l}\text { Nickel. Vet Surg } \\
1998\end{array}$ & \multirow{2}{*}{$\begin{array}{l}\text { Hammock } \\
\text { TVT 5-10\% } \\
\text { Blasen-Perf. }\end{array}$} & \multirow[t]{2}{*}{$>200.000$} \\
\hline 2003 & Urologie & De Leval & TVT-O & $\begin{array}{l}\text { inside-out } \\
\text { Transobturator }\end{array}$ & TOT & & \\
\hline 2006 & & Firma & Pub 2007 & TVT-S & $\mathrm{U}$ oder $\mathrm{H}$ & IUGA 2006 & $>20.000$ \\
\hline
\end{tabular}


2006 wurden die ersten sog. Kurz- oder Minischlingen vorgestellt. Diese Schlingen sollen wie die retropubischen oder transobturatorischen wirken, werden aber eingesetzt, ohne durch das Cavum Retzii oder Foramen obturatum ausgeleitet werden zu müssen. Dadurch sollen intraoperative Komplikationen noch tiefer gehalten werden (wobei aber schon Fallberichte zu Blasenverletzungen und Blutungen erschienen sind). Erste Ergebnisse weisen auf eine 1-Jahreskontinenzrate von $80 \%$ (Meschia 2009, Neumann et al. 2008). Randomisierte Studien liegen nicht vor.

\subsubsection{Indikationsstellung}

Merke: Die operative Behandlung der Belastungsinkontinenz kommt in der Regel nach Ausschöpfen der konservativen Maßnahmen in Frage, wenn die Patientin weiterhin unter der Inkontinenz leidet.

Merke: Dauer der Kontraktion, Dauer der Intervalle, Anzahl der Kontraktionen pro Übungen, Anzahl der Kontraktionen pro Tag sowie die Gesamttrainingsdauer sollten der Patientin als individueller Trainingsplan vorgegeben werden.

Voraussetzung für den Eingriff ist aber auch die Objektivierbarkeit der Belastungsinkontinenz und der präoperative Ausschluss einer Blasenentleerungsstörung. Mitentscheidend für die Wahl der Operationsmethode sind neben einem allfälligen Genitaldeszensus auch die Mobilität der Urethra, die gerade in der Rezidivsituation eingeschränkt sein kann, und der Urethralruheverschlussdruck (hypotone Urethra). Besteht eine Zystozele bei lateralem bzw. paravaginalem Defekt, was häufig mit einer Belastungsinkontinenz vergesellschaftet ist, so kann in dieser Situation die abdominale Kolposuspension helfen. Rezidivsituationen, gerade wenn die Urethra resp. der Blasenhals nicht mehr mobil resp. elevierbar ist, reduzieren die Erfolgsaussichten. Aufgrund der unzureichenden Datenlage kann keine abschließende Empfehlung zur konkreten Indikationsstellung der einzelnen Techniken oder Vorgehen bei gleichzeitig vorliegendem Genitaldeszensus abgegeben werden. Das definitive Vorgehen muss und kann nur gemeinsam mit der Patientin besprochen werden. Zumindest soll primär die bestmögliche Technik un- ter Berücksichtigung des Anästhesierisikos und der Komorbiditäten angewendet werden, da die erneute Chirurgie bei Rezidivinkontinenz resp. voroperierter, hypotoner und hypomobiler Urethra schlechtere Kontinenzresultate aufweist (Comiter 2006).

Drei spezielle Situationen sollen im Folgenden näher erläutert werden.

\section{Vorgehen bei Gentialdeszensus und Belastungs- harninkontinenz}

Diskutabel ist das Vorgehen beim gleichzeitigen Auftreten von Genitaldeszensus und Belastungsinkontinenz, ob ein- oder zweizeitig operiert werden soll. Ist präoperativ eine larvierte Belastungsinkontinenz ausgeschlossen, dann tritt diese auch nicht nach erfolgter Deszensuschirurgie auf (Chaikin et al. 2000). Liegen gleichzeitig eine klinisch relevante Belastungsinkontinenz und ein Genitaldeszensus vor, so richtet sich die Operationsindikation nach der im Vordergrund stehenden Symptomatik bzw. ist ein kombiniertes Vorgehen vertretbar. Im Ab-wägen einer einzeitigen, d. h. simultanen Inkontinenzoperation zur Deszensuschirurgie, gegenüber einer zweizeitigen Vorgehensweise, d. h. Inkontinenzchirurgie erst bei nach erfolgter Deszensuschirurgie demaskierter resp. störender Belastungsinkontinenz, ist die larvierte gegenüber der manifesten Belastungsinkontinenz abzugrenzen und die höhere Rate postoperativer Blasenentleerungsstörungen bei einzeitigem Vorgehen zu berücksichtigen (Fatton et al. 2009). Ein Konsensus fehlt jedoch aufgrund der eingeschränkten Datenlage. Bei larvierter Belastungsinkontinenz ist die konkomitante TVT-Einlage der alleinigen Diaphragmaplastik überlegen (objektive Kontinenzrate nach zwei Jahren 92 vs. $56 \%$ ), ohne die Komplikationsrate statistisch signifikant zu erhöhen, wie eine prospektiv randomisierte Studie zeigt (Meschia 2004). Bei 87 Patientinnen mit Deszensuschirurgie und gleichzeitiger TVT-Einlage (Gruppe I) zeigte der Vergleich mit 94 Frauen, bei denen die TVT-Einlage erst drei Monate nach erfolgter Deszensuschirurgie geplant war (Gruppe II), dass $44 \%$ der Gruppe II nach drei Monaten keine Schlingenoperation benötigten, da $29 \%$ kontinent wurden und weitere $15 \%$ bei nur geringem Urinverlust keine Schlingeneinlage wollten (Borstad et al. 2008). Auch bei Diaphragmaplastik konnte eine Wiederherstellung der Kontinenz in knapp $60 \%$ beobachtet werden, wobei die Diaphragmaplastik nicht als Inkonti- 
nenzoperation angesehen werden sollte (Glazener et al. 2001). Beim simultanen Eingriff wird also nicht die erzielbare Kontinenz in Frage, sondern die möglicherweise erhöhte Komplikationsrate und höhere Rate postoperativer Blasenentleerungsstörungen insbesondere nach Korrektur des vorderen Kompartimentes in den Vordergrund gestellt (Anger et al. 2008). Entscheidet man sich gemeinsam mit der Patientin für das zweizeitige Vorgehen, dann empfiehlt sich der allfällige Inkontinenzeingriff drei Monate nach der Deszensuschirurgie, wenn sich anatomisch stabile Verhältnisse ergeben haben. Im klinischen Alltag wird man für eine jüngere Frau mit moderatem Deszensus und bereits manifester Belastungsinkontinenz ein kombiniertes Vorgehen und für eine ältere Frau mit hochgradigem Prolaps und möglicher larvierter Inkontinenz ein zweizeitiges Vorgehen erwägen.

\section{Hypermobile versus hypotone Urethra}

Merke: Die "klassische" Indikationsstellung für die moderne suburethrale Schlinge wie das TVT ist die Belastungsinkontinenz bei (hyper) mobiler Urethra.

Verschiedene Autoren konnten zeigen, dass bei hypo- bis amobiler Urethra, die u. a. bei Rezidivinkontinenz auftritt, oder bei hypotoner Urethra (maximaler Urethralruheverschlussdruck $<20 \mathrm{~cm}$ $\mathrm{H}_{2} \mathrm{O}$ ) die Erfolgschancen reduziert sind. Rezidivinkontinenz und Alter sind entsprechende Risikofaktoren. So nimmt mit zunehmendem Alter der maximale Urethralruheverschlussdruck physiologischerweise aufgrund der kontinuierlichen Abnahme der Muskelfasern ab (Perucchini et al. 2002). Das gleichzeitige Vorliegen einer hypotonen Urethra und hypo- oder amobilen Urethra wird auch als intrinsische Sphinkterinsuffizienz bezeichnet. TVT in der Rezidivsituation zeigt eine Erfolgsrate von $90 \%$ bei genügend mobiler Urethra, aber nur $33 \%$ bei fixierter Urethra (Liapis et al. 2004). Dieselben Autoren zeigten auch für die hypotone Urethra eine Heilungsrate von $73 \%$ und deutliche Verbesserung der Inkontinenzsituation bei weiteren $9 \%$ und folgerten eine zufriedenstellende Kontinenzrate auch in der hypotonen Situation (Liapis et al. 2004). Unter Berücksichtigung der urethralen Hypermobilität ergab sich sogar eine Kontinenz und Besserung in $87 \%$ resp. 7 \%, wäh- rend die fixierte Urethra eine Versagerquote von $57 \%$ aufwies. Auch nach vier Jahren fanden andere Autoren nach TVT-Einlage bei 34 Frauen in der Rezidivsituation nach traditioneller Inkontinenzchirurgie Heilungsraten von $82 \%$ und eine Verbesserung in $9 \%$ (Rezapour et al. 2001) und konnten in einer weiteren Studie ebenfalls nach vier Jahren Heilungsraten von $74 \%$ und Besserung bei $12 \%$ für 49 Frauen mit hypotoner Urethra zeigen (Rezapour et al. 2001), wobei die Frauen mit persistierender Inkontinenz über 70-jährig waren und einen Urethraldruck von unter $10 \mathrm{~cm} \mathrm{H} 2 \mathrm{O}$ aufwiesen. Miller (2006) fand für das transobturatorische TOT im Vergleich zum retropubischen TVT ein 5,9-fach höheres Risiko für eine postoperative Inkontinenz nach drei Monaten bei hypotoner (cut-off $42 \mathrm{~cm} \mathrm{H} 2 \mathrm{O}$ ), allerdings mobiler Urethra. Gerade bei älteren Frauen mit ausgesprochen hypotoner und immobiler Urethra ist von einem reduzierten Operationserfolg auszugehen. Von den aktuellen Inkontinenzschlingen ist derzeit das retropubische TVT am besten untersucht, weswegen wir diese Schlinge auch für solche Spezialsituationen bevorzugen.

\section{Adipositas}

Verschiedene Autoren konnten zeigen, dass Adipositas das Outcome von Inkontinenzoperationen nicht verschlechtert (Lovatsis et al. 2003, Rafii et al. 2003, Zivkovic et al. 1999).

\section{Mischharninkontinenz}

Zur Indikation, Inkontinenzchirurgie und Erfolg bei Patientinnen mit Mischsymptomatik oder Mischinkontinenz sei auf das Kapitel 9 verwiesen.

\subsubsection{Operative Prinzipien}

Der operative Zugang kann von vaginal, abdominal oder laparoskopisch, kombiniert vagino-abdominal oder transurethral erfolgen. Dabei ist der minimalinvasive Ansatz der transurethrale zur periresp. transurethralen Injektion von sog. Bulking agents, gefolgt von den modernen suburethralen Schlingen.

Zu den vaginalen Operationsverfahren zählen die früher verwendeten Kelly-Nähte, die vordere Raffung (die allerdings keine Inkontinenzoperation ist), die Schlingen mittels Fascia lata oder Dura mater, die heute verbreiteten und mittler- 
Tab. 16.4 Differentialindikation resp. Vorzug für eine Route. $\mathrm{X}=$ indizierbar; ORG = nach Originalmethode

\begin{tabular}{|c|c|c|c|}
\hline & Indikation & Retropubisch & Transobturatorisch \\
\hline \multirow[t]{2}{*}{ Anästhesie } & Lokalanästhesie & ORG & $X$ \\
\hline & Teil- oder Vollnarkose & $(\mathrm{X})$ & ORG \\
\hline Lagerung & Hüftbeschwerden & $x$ & \\
\hline Urethra & hypotone Urethra & $x$ & \\
\hline Anatomie & enger Introitus & $(\mathrm{X})$ & $x$ \\
\hline \multirow[t]{2}{*}{ Voroperationen } & vernarbtes Cavum retzii & $(\mathrm{X})$ & $x$ \\
\hline & (Inguinal-)Hernie & & $x$ \\
\hline BMI & Adipositas & $x$ & \\
\hline \multirow[t]{2}{*}{ Integument } & suprasymphysäre Affektion & & $x$ \\
\hline & ischiocrurale Affektion & $x$ & \\
\hline Hämatologie & Blutungsrisiko & $(\mathrm{X})$ & \\
\hline
\end{tabular}

weile als Goldstandard angesehenen modernen spannungsfreien suburethralen Schlingenoperationen. Zu den abdominalen Suspensionsverfahren gehören die offene Kolposuspension nach Marshall-Marchetti-Krantz resp. deren Modifikation und Goldstandard nach Burch und später Cowan. Die Kolposuspension wird seit den 90er-Jahren auch laparoskopisch durchgeführt (Dean et al. 2006).

\section{Differentialindikation}

Prinzipiell kann die retropubische Schlinge immer eingesetzt werden, sofern keine absolute Kontraindikation dagegen spricht $(\checkmark$ Tab. 16.4). Retropubisch kann gerade auch bei älteren Frauen mit schlecht beweglichen oder voroperierten Hüften in flacher Oberschenkellagerung in Lokalanästhesie vorgegangen werden.

\subsection{Operatives Vorgehen (ausgewählte Verfahren)}

Die modernen suburethralen Schlingen haben das Gros der älteren Inkontinenztechniken faktisch verdrängt.

Nachdem heute die spannungsfreie suburethrale Schlingentechnik in Europa quasi als Standard gilt, soll im Folgenden auf heute noch gebräuchliche Techniken mit Schwergewicht auf das TVT eingegangen werden.

Alle Inkontinenzeingriffe erfolgen unter perioperativer Antibiotikaprophylaxe, z. B. einmalig bei Einleitung Cefazolin.

\subsubsection{Spannungsfreie vaginale Schlingen- techniken (retropubisches Tension-free Vaginal Tape TVT nach Ulmsten und Petros 1995, transobturatorische Route out- side-in TOT nach Delorme 2001 und inside-out TVT-O nach De Le- val 2003)}

Indikation

Wahrscheinlich bei prämenopausalen Patientinnen hinsichtlich allfälligen Spätarrosionen des alloplastischen Materials wenig problematisch, aber auch noch nicht definitiv beantwortet. Der Eingriff in Lokalanästhesie und Analgosedation ist gerade bei eingeschränkter Narkosefähigkeit von Vorteil

\section{Retropubisches TVT nach Ulmsten und Petros 1995}

Der Eingriff erfolgt üblicherweise in Lokalanästhesie

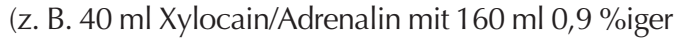
$\mathrm{NaCl}$-Lösung verdünnt) und Analgosedation. Die Beine werden flach gelagert (Oberschenkel 40-60 flektiert). Die Harnblase wird katheterisiert. Knapp oberhalb der Symphyse werden die Austrittstellen je $2 \mathrm{~cm}$ lateral der Mittellinie markiert. Hier werden je $5 \mathrm{ml}$ des Lokalanästhetikums subkutan, dann je $10 \mathrm{ml}$ in die Bauchdecke, anschließend je $20 \mathrm{ml}$ ins Cavum Retzii injiziert. Ein transurethraler Dauerkatheter Ch 18 wird mit Führungsstab eingeführt. Von vaginal werden nun $5 \mathrm{ml}$ Lokalanästhetikum suburethral, dann nach jeweiligem Abdrängen der Urethra mittels Führungsstab beidseits je $20 \mathrm{ml}$ ins 
Diaphragma urogenitale injiziert. Midurethral, $1 \mathrm{~cm}$ vom Meatus externus entfernt beginnend, erfolgt die 10-15 mm lange suburethrale Kolpotomie in Mittellinie. Unter erneutem Wegdrängen der Urethra dissezieren wir beidseits stumpf bis zum Diaphragma urogenitale. Nun wird nach entsprechendem Wegdrängen der Urethra das ca. $10 \mathrm{~mm}$ breite, makroporöse (> 75 Mikron) monofilamentäre Kunststoffband aus Polypropylen (Prolene), das in einer Plastikhülle liegt, mit einem $5 \mathrm{~mm}$ dicken Tunnelierer (Spezialnadel) retropubisch unter gleichzeitiger zystoskopischer Kontrolle hochgeführt (die scharfe Plastikhülle kann die Blase dabei verletzen) und an vormarkierter Stelle über die zwei suprasymphysären Stichinzisionen ausgeleitet ( Abb. 16.3). Zur Anpassung der Bandspannung und intraoperativen Erfolgskontrolle soll die Patientin husten: Das Band wird soweit angezogen, dass es noch spannungsfrei unter der Harnröhre liegt und beim Husten gerade noch tropfenweise Urin abgeht. Das Band soll weder eine Elevation noch Obstruktion bewirken, sondern lediglich als Matrix für eine Neufixation der Urethra durch Einsprossung von Fibroblasten dienen. Jetzt können die $\mathrm{Na}$ deln vom Band abgetrennt und die Plastikhülle unter Gegenhalten des Bandes suburethral unter Beachtung der Spannungsfreiheit abgezogen werden. Die suprasymphysären Enden werden gekürzt

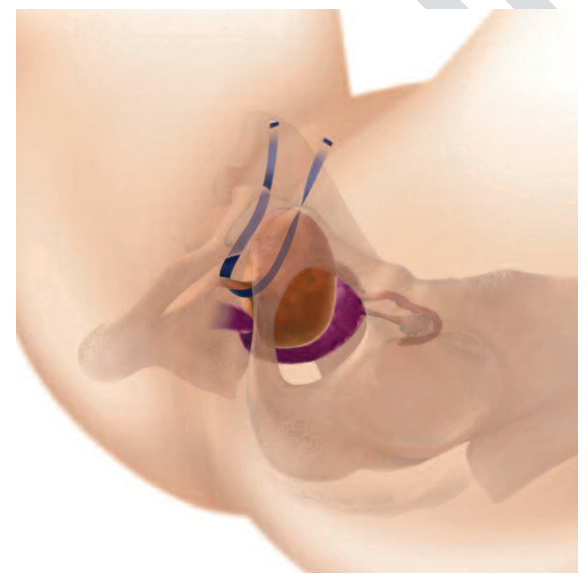

Abb. 16.3 Prinzip der retropubischen Route. Das Band wird über eine suburethrale Kolpotomie beidseits der Harnröhre nach Abdrängen derselben hinter der Symphyse hochgeführt und suprasymphysär ausgeleitet. Die Passage durch das kleine Becken erklärt das mögliche Komplikationsmuster. und unter der Haut versenkt. Das Proleneband muss weder speziell in der Bauchwand noch suburethral fixiert werden, da es durch seine geflochtene Netzstruktur mit kleinen "Widerhäkchen" selbsthaftend im Gewebe verbleibt. Deshalb muss es in einer Plastikhülle durchgezogen werden. Kolpotomie und Hautinzision werden verschlossen. Nun wird die Blase katheterisiert, wobei in der Regel kein Dauerkatheter gelegt werden muss.

\section{Transobturatorische Verfahren}

Zur Vermeidung der aufgrund der Passage durch das kleine Becken bedingten Komplikationen der retropubischen Verfahren beschrieb Delorme 2001 den transobturatorischen Zugang zur Platzierung eines suburethralen Bandes ( $\triangleright$ Abb. 16.4). Da die Bänder unter die endopelvine Faszie gelegt werden und das Cavum Retzii oder das kleine Becken nicht passieren, sind Blasenverletzungen nicht zu erwarten und eine Zystoskopie meist nicht notwendig. Inzwischen sind zahlreiche Verfahren verschiedener Firmen auf dem Markt (und die ursprünglichen Bänder bereits vom Markt genommen). Grundsätzlich wird das Band entweder von außen nach innen ("outside in") oder von innen nach außen (inside out“), jeweils mit einer speziellen helixartigen Nadel, geführt. Die von Delorme

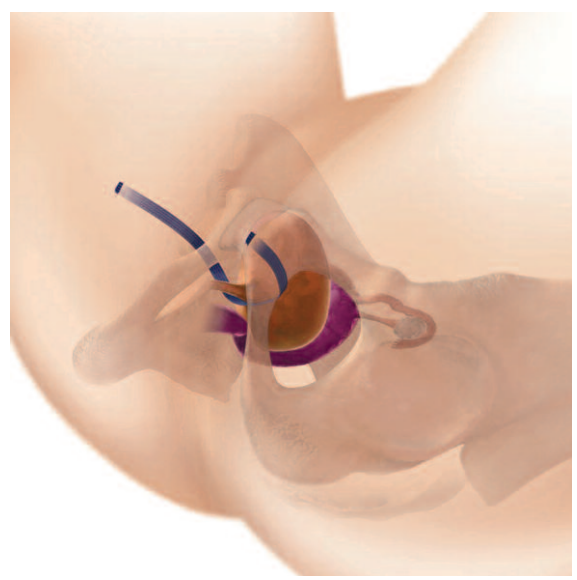

Abb. 16.4 Prinzip der transobturatorischen Bandroute. Wie bei der retropubischen Route wird das Band über eine suburethrale Kolpotomie eingeführt, dann aber beidseits lateral durch die Foramina obturatoria ausgeleitet (inside-out). Die Outside-in-Technik führt entsprechend das Band von außen ischiocrural durch die Foramina obturatoria nach suburethral. 
Tab. 16.5 Operative Unterschiede der einzelnen Schlingen. SSL = Steinschnittlage; LA = Lokalanästhesie; SA = Spinal-/Regionalanästhesie; ITN = Intubations-/Vollnarkose

\begin{tabular}{|c|c|c|c|}
\hline Produkt & TVT & TOT & TVT-O \\
\hline Zugang und Richtung & $\begin{array}{l}\text { von vaginal retropubisch } \\
\text { nach suprasymphysär aufwärts }\end{array}$ & $\begin{array}{l}\text { von ischiocrural zu den } \\
\text { Foramina obturatoria } \\
\text { (outside-in) }\end{array}$ & $\begin{array}{l}\text { von vaginal zu den Foramina } \\
\text { transobturatoria (inside-out) }\end{array}$ \\
\hline Lagerung & $\mathrm{SSL}<60^{\circ}$ & SSL $120^{\circ}$ & \\
\hline Anästhesie & LA (SA, ITN) & SA, ITN (LA) & \\
\hline Zystoskopie & obligat & (ja) & (ja) \\
\hline Hustentest & ja & nein & nein \\
\hline Dauerkatheter & nein & (1 Tag) & \\
\hline Zusatzeingriffe & möglich & möglich & \\
\hline
\end{tabular}

Tab. 16.6 Anatomische Unterschiede der beiden transobturatorischen Routen outside-in resp. inside-out

\begin{tabular}{|c|c|c|c|c|}
\hline Autor & $\begin{array}{l}\text { Bänder } \\
\text { (n) }\end{array}$ & $\begin{array}{l}\text { outside-in } \\
\text { TOT }\end{array}$ & $\begin{array}{l}\text { inside-out } \\
\text { TVT-O }\end{array}$ & Favorisierung \\
\hline $\begin{array}{l}\text { Bonnet } \\
2005\end{array}$ & 12 & & $\begin{array}{l}\text { Der kürzeste Abstand des Bandes } \\
\text { zum N. obturatorius beträgt } 22 \mathrm{~mm} \text {. }\end{array}$ & \\
\hline $\begin{array}{l}\text { Delmas } \\
2005\end{array}$ & 10 & $\begin{array}{l}\text { keine Gefäß- oder Nervenverlet- } \\
\text { zung bei korrekter Anwendung }\end{array}$ & & \\
\hline $\begin{array}{l}\text { Spinosa } \\
2005\end{array}$ & 7 & $\begin{array}{l}\text { Gefäßbündel }>3 \mathrm{~cm} \text { und hinterer } \\
\text { Ast } N \text {. obturatorius }>2,1 \mathrm{~cm} \text { ent- } \\
\text { fernt }\end{array}$ & $\begin{array}{l}\text { Gefäßbündel } 4 \times<1 \mathrm{~cm}, \\
1 \times>1 \mathrm{~cm}, 1 \times \text { verletzt hinterer Ast } \\
\text { N. obturatoriuas } 0,9 \mathrm{~cm}, 1 \times \text { verletzt }\end{array}$ & TOT \\
\hline $\begin{array}{l}\text { Reisenauer } \\
2006\end{array}$ & 5 & $\begin{array}{l}\text { Passiert nie das Cavum Retzii. } \\
2 \text { bis } 2,8 \mathrm{~cm} \text { zu Ramus anterior des } \\
\text { N. obturatorius an der Oberfläche } \\
\text { des M. adductor brevis. 2,0 bis } \\
2,9 \mathrm{~cm} \text { im Foramen obturatum zu } \\
\text { Nerven/A. obturatoria. Deren vor- } \\
\text { derer Ast verläuft geschützt an der } \\
\text { äußeren Kante des unteren Scham- } \\
\text { beinastes. }\end{array}$ & & \\
\hline $\begin{array}{l}\text { Achtari } \\
2006\end{array}$ & 10 & $\begin{array}{l}\text { N. clitoridis dorsalis verläuft unter } \\
\text { dem Schambein 14,3 } \pm 4,7 \text { mm } \\
\text { von der Mittellinie. Bänder ähnlich } \\
\text { weit vom Nerv entfernt. TOT vom } \\
\text { Obturatorkanal 40,1 mm }\end{array}$ & $\begin{array}{l}\text { N. clitoridis dorsalis verläuft unter } \\
\text { dem Schambein } 14,3 \pm 4,7 \mathrm{~mm} \\
\text { von der Mittellinie. Bänder } \\
\text { ähnlich weit vom Nerv entfernt. } \\
\text { TVT-O vom Obturatorkanal } 19 \mathrm{~mm}\end{array}$ & TOT \\
\hline $\begin{array}{l}\text { Hinoul } \\
2007\end{array}$ & 6 & & $\begin{array}{l}0,7-2,0 \mathrm{~cm} \text { vom Obturatorkanal, } \\
0,5-2,0 \mathrm{~cm} \text { vom N. obturatorius } \\
\text { (vorderer Ast), } 0,1-1,4 \mathrm{~cm} \\
\text { (hinterer Ast) }\end{array}$ & \\
\hline $\begin{array}{l}\text { Zahn } \\
2007\end{array}$ & 7 & $\begin{array}{l}\text { weiter vom Obturatorkanal }(2,3 \mathrm{~cm}) \\
\text { und näher zum unteren Schambein- } \\
\text { ast }(0,04 \mathrm{~cm}) \\
\text { Abstände links größer }\end{array}$ & $\begin{array}{l}\text { näher zum Obturatorkanal }(1,3 \mathrm{~cm}) \\
\text { und weiter vom unteren Scham- } \\
\text { beinast }(0,39 \mathrm{~cm}) \\
\text { Abstände links größer }\end{array}$ & TOT \\
\hline $\begin{array}{l}\text { Ottem } \\
2007\end{array}$ & 9 & $\begin{array}{l}\text { Vene in } 78 \% \text { medial, Arterie in } 22 \% \text {. } \\
\text { Nerv immer lateral. Venen- }(22 \%) \\
\text { und Arterien- }(17 \%) \text { Äste der Obtura- } \\
\text { torgefäße überqueren die Obturator- } \\
\text { membran nach medial Richtung } \\
\text { Trokarplatzierung. } 30 \text { mm Durch- } \\
\text { schnittsabstand von optimaler Trokar- } \\
\text { platzierung zu Obturatorkanal. }\end{array}$ & & \\
\hline
\end{tabular}


beschriebene Technik war von außen nach innen; die Möglichkeit, von innen (vaginal) nach außen zu stechen, wurde von De Leval 2003 beschrieben.

Im Unterschied zum retropubischen Verfahren sollen beim transobturatorischen Vorgehen die Beine in den Hüften auf etwa $120^{\circ}$ überflektiert werden, um die Anatomie mit Zugang zu den Foramina obturatoria korrekt zu präsentieren. - Tab. 16.5 veranschaulicht die operativ-technischen Unterschiede der beiden Routen. Nach Durchsicht der acht anatomischen Untersuchungen an Leichen zu den unterschiedlichen transobturatorischen Zugängen scheint die Outside-inTechnik favorisiert zu werden, wobei die klinische und insbesondere die eigene Erfahrung diesbezüglich (noch) keine Aussage zulässt ( Tab. 16.6).

Intraoperativ soll auf die spannungsfreie Bandlage geachtet werden: Das Band wird in der Mitte mit einer Babcock-Klemme gefasst, wodurch eine etwa $5 \mathrm{~mm}$ lange Schlaufe entsteht. Dann wird das Band an die Urethra herangeführt, bis die Babcock-Klemme der Urethra aufliegt. Jetzt wird die Klemme gelöst, und das Band liegt nun spannungsfrei der Urethra an. Besteht eine offensichtliche "Luftschlaufe", wird das Band noch vorsichtig angezogen. Verschwindet das Band hinter der Kolpotomie und muss gewissermaßen mühselig aufgesucht werden, ist es wahrscheinlich zu straff und sollte etwas gelockert werden. Es empfiehlt sich, das Band lateral und möglichst hoch mit Péan-Klemmen zu fassen und zurückzuziehen, damit das Band suburethral nicht durch den direkten Zug ausgedünnt wird (je nach Band- resp. Produkteigenschaften unterschiedlich). Das korrekt sitzende Band liegt flach in seiner vollen Breite suburethral auf, ist gut einsehbar und lässt sich mit einer breiten, z. B. der Metzenbaumschere, bequem unterfahren. Wenn jetzt noch beim Hustentest einige Tropfen Urin abgehen, sollte das Band korrekt liegen, ohne dass ein Harnverhalt zu erwarten ist.

\section{Erfolg und Misserfolg}

Das TVT zeigt vergleichbar gute Langzeit-Kontinenzraten von über $80 \%$ wie der bisherige Standard, die Kolposuspension nach Burch (Nilsson et al. 2008, Ward et al. 2008). Transobturatorische Bänder erzielen vergleichbare Kontinenzraten von $87 \%$ wie das TVT (Latthe et al. 2007, Sung et al. 2007). Die Erfolgsrate nimmt bei Mischinkontinenz oder bei präoperativer Trichterbildung am Blasen- hals ab. Ebenso ist im Alter von einer etwas geringeren Erfolgsrate auszugehen (Rezapour et al. 2001). Dies ist präoperativ mit der Patientin zu besprechen. Häufig tritt gleichzeitig eine überaktive Blase auf, und durch den hypotonen Detrusormuskel können postoperativ Blasenentleerungsstörungen vorkommen. Risikofaktoren für die Entwicklung einer Rezidivinkontinenz nach Schlingeneinlage scheinen ein gleichzeitiger Deszensuseingriff oder ein präoperative anticholinerge Medikation zu sein. Zunehmendes Alter ist spezifisch mit einem Wiederauftreten von Symptomen der Belastungsinkontinenz assoziiert (Barber et al. 2008).

\section{Komplikationen und deren Management}

Unterschieden wird zwischen intraoperativen Komplikationen (z. B. Blutung, Organverletzung), unmittelbar postoperativen Komplikationen (z. B. Blasenentleerungsstörung) sowie den längerfristigen postoperativen Problemen.

Intraoperative Komplikationen. Die Blasenperforation sollte durch die intraoperative Zystoskopie erkannt werden und ist vergleichsweise harmlos. Prädilektionsstellen sind an der Seitenwand bei 10 und 2 Uhr. Die Nadel wird zurückgezogen und neu gesetzt. Unter prophylaktischer Antibiotikagabe und Belassen eines transurethralen Blasenverweilkatheters für einen Tag heilt die Perforation komplikationslos ab. Eine übersehene Blasenperforation mit verbleibendem intravesikalem Mesh führt zu rezidivierenden Harnweginfektionen, Konkrementbildung und Drangsymptomatik. Intraoperative Blutungen kommen meist mit einer kurzfristigen Tamponade zum Stillstand. Mit retropubischen Verfahren sind Verletzungen großer Gefäße mit starken Blutungen selten, aber beschrieben (Kölle et al. 2007).

Postoperative Komplikationen. Retropubisch: Hämatome Darmverletzungen. TO Verfahren: bei den transobturatorischen Bändern eher Schmerzen im Leistenkanal und Oberschenkel, Vaginalperforationen oder Erosionen (Latthe et al. 2007, Sung et al. 2007). Zunehmend wurden auch Abszesse nach transobturatorischer Bandeinlage beschrieben, möglicherweise gefördert durch das Material gewisser Bänder, die bereits vom Markt genommen worden sind, und das Passieren der Adduktorenmuskeln bei den transobturatorischen Bändern, im Gegensatz zum sehnigen Ansatz des M. rectus abdominis bei den retropubischen (Tamus- 
Tab. 16.7 Komplikationen bei retropubischen und transobturatorischen Schlingen. $\mathrm{A}=\mathrm{Abszess} ; \mathrm{B}=\mathrm{Blutung}$; $\mathrm{BES}=$ Blasenentleerungsstörung; $\mathrm{E}=$ Erosion; $\mathrm{P}=$ Perforation; $\mathrm{R}=($ Harn-) Retention

\begin{tabular}{|c|c|c|c|c|}
\hline Autor & Band & Follow-up & Kont. & Anmerkung \\
\hline $\begin{array}{l}\text { Costa } \\
2004\end{array}$ & 183 Uratape & 7 Monate & & $3,3 \%$ R., 3,6 \% E \\
\hline $\begin{array}{l}\text { Minaglia } \\
2004\end{array}$ & 61 Mentor & k. A. & & $5 \% P$ \\
\hline $\begin{array}{l}\text { But } \\
2005\end{array}$ & 30 Monarc & 6 Wochen & & $7 \% \mathrm{E}$ \\
\hline $\begin{array}{l}\text { Domingo } \\
2005\end{array}$ & 64 Ura/Obtape & 10 Monate & & $14 \% \mathrm{E}, 1$ mit $\mathrm{A}$ \\
\hline $\begin{array}{l}\text { Enzelsberger } \\
2005\end{array}$ & $\begin{array}{l}54 \text { TVT } \\
56 \text { TOT }\end{array}$ & 15 Monate & $86 \%$ vs. $84 \%$ & $\begin{array}{l}\text { OP } 25 \text { vs. } 15 \text { Min. } \\
1 \text { vs. } 1 \text { E. TVT 7,6 \% } \\
\text { Blasen-P. BES 7,6 \% } \\
\text { vs. } 5,6 \%\end{array}$ \\
\hline $\begin{array}{l}\text { Fischer } \\
2005\end{array}$ & $\begin{array}{l}220 \text { TVT } \\
220 \text { TOT }\end{array}$ & >12 Monate & $76 \%$ vs. $81 \%$ & $\begin{array}{l}\text { Spaltung } 4 \text { vs. } 2 \% \\
\text { Blasen-P } 4,5 \text { vs. } 0,5 \%\end{array}$ \\
\hline $\begin{array}{l}\text { Krauth } \\
2005\end{array}$ & 604 I-Stop & 131 nach 1 Jahr & & $\begin{array}{l}2 \text { gelockert/durch- } \\
\text { trennt. } 0,3 \% \text { P. 1,1\% B }\end{array}$ \\
\hline $\begin{array}{l}\text { Siegel } \\
2005\end{array}$ & 30 Obtape & 12 Monate & & $20 \%$ E (1 Obt.A) \\
\hline $\begin{array}{l}\text { Debodinance } \\
2006\end{array}$ & $\begin{array}{l}50 \text { Monarc } \\
50 \text { TVT-O }\end{array}$ & 12 Monate & $90 \%$ vs. $94 \%$ & $\begin{array}{l}\text { Mon } 1 \text { vag } P \text {, } \\
1 \text { Bein-Sz n. } 1 \text { Jahr } \\
54 \% \text { sex. aktiv, oB }\end{array}$ \\
\hline $\begin{array}{l}\text { Deval } \\
2006\end{array}$ & 129 Obtape & 17 Monate & & $\begin{array}{l}\text { 1,5 \% justiert. } 5 \% \\
\text { E. } 6 \text { Bänder entfernt }\end{array}$ \\
\hline $\begin{array}{l}\text { Dobson } \\
2007\end{array}$ & 52 Obtap & 12 Monate & & E $15 \%, 1 \mathrm{~A}$ \\
\hline $\begin{array}{l}\text { Falkert } \\
2007\end{array}$ & $\begin{array}{l}56 \text { TVT } \\
49 \text { TOT }\end{array}$ & k. A. & $90 \%$ vs. $96 \%$ & R 23 vs. $2 \%$ \\
\hline $\begin{array}{l}\text { Kyu-Sung } \\
2007\end{array}$ & $\begin{array}{l}60 \text { TVT } \\
60 \text { TVT-O }\end{array}$ & 13 Monate & $87 \%$ vs. $87 \%$ & 4 TVT-O De-novo-Urge \\
\hline
\end{tabular}

sino et al. 2007). Als schwerwiegende Komplikationen sind nekrotisierende Faszitis, Osteomyelitis und Osteonekrose nach transobturatorischer Bandeinlage beschrieben. Banderosionen treten in 3,6-20\% auf, und Miktionsstörungen werden in $5 \%$ beschrieben ( Tab. 16.7).

Die Banderosionsrate hängt aber nicht nur von der Route ab, sondern auch vom Material. Gerade mit mikroporösen oder multifilamentären Netzen wurden sehr schlechte Erfahrungen gemacht. Die Textur der heutzutage eingesetzten makroporösen und monofilamentären Schlingen sorgt für das Ausbleiben von Bandinfektionen bzw. -abstoßungen. Allerdings sind auch Späterosionen des Bandes in die Blase nach fünf Jahren beschrieben (Quereux et al. 2005).
Postoperative Blasenentleerungsstörungen. Die meisten Frauen nach retropubischen oder transobturatorischen Bändern können innerhalb eines Tages restharnfrei $(<100 \mathrm{ml})$ miktionieren. Schwellungen oder Hämatome periurethral oder im Cavum Retzii sowie die Lokalanästhesie können in den ersten Tagen Blasenentleerungsstörungen von erhöhter Restharnmenge bis zum kompletten Harnverhalt verursachen, Operationswürdige Blasenentleerungsstörungen treten in etwa $3 \%$ auf (Tamussino et al. 2001, 2007). Persistiert die Blasenentleerungsstörung mit Restharnmengen deutlich über 150 ml bei ungenügender Spontanmiktion, kann die Bandlockerung versucht werden, indem mit einem Hegarstift der Größe 7 oder 8 in die Urethra eingegangen und diese mit vorsichti- 
gen ruckartigen Bewegungen nach dorsal gedrückt wird. Dieses Vorgehen wird gut toleriert, bei Bedarf kann eine halbe Stunde zuvor Tramadol verabreicht werden. Hilft diese Maßnahme nicht weiter, stehen zwei Optionen offen. Entweder wird in Lokalanästhesie oder Kurznarkose die Kolpotomie innerhalb der ersten sieben Tage wieder eröffnet, um dann das Band lateral möglichst hoch mit je einer (stumpfen) Péan-Klemme zu fassen, um einige Millimeter nach unten zu ziehen und zu lockern, oder das Band wird bei Persistenz der Blasenentleerungsstörung erst nach Einheilung und Vernarbung zwei bis drei Monate postoperativ gespalten oder teilexzidiert. Letztgenanntes Vorgehen bedingt den Selbstkatheterismus oder Einlage eines Dauerkatheters und viel Geduld, doch kann in etwa $70 \%$ nach Bandspaltung mit einer erhaltenen Kontinenz gerechnet werden (Klutke et al. 2001). Andererseits erlaubt das unmittelbare postoperative Vorgehen den Erhalt des Bandes und Kontinenzmechanismus sowie die rasche Behebung der Obstruktion und entsprechend Rückkehr zur Normalität.

Die klinische Untersuchung und Bougierung der Harnröhre mit sogenannten Bougie-à-boules, mit denen beim Rückzug ein allfälliger Widerstand des Bandes besser erspürt werden kann (mit normalen Hegarstiften wird der bandbedingte Widerstand wie mit einem Pflug durchbrochen und bleibt unbemerkt) sowie die Perinealsonographie zur Beurteilung der Bandposition (nahe am Blasenhals? nahe zur Urethra? Kinking der Urethra) in Ruhe und unter Belastung sowie zum Ausschluss eines klinisch relevanten Hämatomes hilft für das Verständnis weiter, wobei ein persistierender Harnverhalt letztlich durch das Band bedingt ist und behoben werden sollte. Miktionsstörungen wie verlängerte oder stakkatoförmige Miktion in 16 \% können die Lebensqualität beeinflussen (Vervest et al. 2007).

De-novo-Urgency, wahrscheinlich verursacht durch eine milde Obstruktion der Urethra und urethrale Reizung durch das Band, tritt beim TVT in 6-25\% und bei den transobturatorischen Bändern in $0-16 \%$ auf, wobei die Unterschiede nicht signifikant scheinen (Daneshgari et al. 2008). Tatsächlich finden wir in unserem Kollektiv bandunabhängig eine Reduktion des freien maximalen Urinflusses um $28 \%$ von 29 auf $21 \mathrm{ml} / \mathrm{s}$. Sofern eine operationswürdige Blasenentleerungsstörung ausgeschlossen ist, kann die De-novo-Urgency zu- nächst konservativ mit Beckenbodenrehabilitation, Anticholinergika oder dann mittels intravesikaler Injektion von Botulinumneurotoxin angegangen werden.

Merke: Banderosionen sind beim TVT selten $(1 \%)$ und treten meist suburethral auf, sind aber umso häufiger bei den transobturatorischen Bändern (10 \%).

Dies steht möglicherweise mit der größeren resp. langstreckigeren Kontaktfläche der transobturatorischen Bänder zur Vagina und, da die Erosionen typischerweise in den lateralen Sulci auftreten, mit hohen Sulci im Zusammenhang. Atrophe Vaginalverhältnisse, mitunter bedingt durch Kortikosteroide oder Methothrexat-Therapie bei rheumatischen Begleiterkrankungen, begünstigen das Auftreten von Erosionen. Von den echten (de novo) Erosionen sind die intraoperativ unentdeckt gebliebenen Perforationen zu unterscheiden. Erosionen können jederzeit auftreten, werden aber aus eigener Erfahrung typischerweise ab sechs Wochen postoperativ bemerkt. Die Symptome reichen von Beschwerdefreiheit (quasi Zufallsbefund) über vermehrten vaginalen Ausfluss oder Blutung bis hin zu Fremdkörpergefühl, Geschlechtsverkehr assoziierten Beschwerden ( $\triangleright$ Kap. 24.2.3) oder gar Hispareunie, worunter wir bei diesem angelsächsischen Neologismus die entsprechenden Beschwerden beim Sexualpartner umschreiben. Prinzipiell können Erosionen zunächst konservativ mit intensiver Östrogenisierung über zwei bis drei Monate angegangen werden. Während beim TVT die suburethrale Erosion nach Wundsäuberung und Anfrischen der Vaginalränder unter Antibiotikaprophylaxe meist nach Übernähen behoben ist, können bei den transobturatorischen Bändern die Erosionen wieder auftreten, zumal das Band quer durch den Sulcus zieht und erneut erodiert. Entsprechend muss hier die Teilexzision des Bandes indiziert und bei erneuter Inkontinenz ein zweites Band eingelegt werden.

Merke: Geschlechtsverkehr assoziierte Beschwerden sind eine typische Erscheinung nach transobturatorischer Bandeinlage in bis zu $16 \%$ der sexuell aktiven Frauen, kommen aber auch in etwa $2 \%$ beim TVT vor. 
Zum Beschwerdemuster zählen nebst Beschwerden bei der Penetration Schmerzen bei Ad- oder Abduktion der Oberschenkel, auch unabhängig vom Geschlechtsverkehr, Schmerzen im Bereich der ischiocruralen Bandaustrittstellen, aber auch die sogenannte Hispareunie (Schmerzen des Mannes durch Friktion eines in die Vagina erodierten synthetischen Gewebes). Mitunter ist dies das einzig angegebene Symptom bei sonst beschwerdefreier Patientin. Ultima Ratio in der Behandlung ist die Bandteilexzision und bei erneuter Inkontinenz die Einlage eines (retropubischen) Bandes.

Selbstverständlich ist vor allen operativen Maßnahmen zur Behebung der Komplikationen, seien es nun Blasenentleerungsstörungen, Banderosionen oder Geschlechtsverkehr assoziierte Beschwerden, die Möglichkeit der Banddurchtrennung resp. Teilexzision zu besprechen, mit dem Risiko des Wiederauftretens einer Inkontinenz. Immer wieder trifft man auf Patientinnen, denen die erlangte Kontinenz wichtiger ist als die Behebung der Blasenentleerungsstörung oder die die Erosion nicht spüren resp. nur ihr Sexualpartner.

\subsubsection{Abdominale Kolposuspension (retropubische Urethropexie) nach Burch 1961 (Modifikation nach Cowan 1979)}

\section{Indikation}

- Belastungsinkontinenz bei Traktionszystozele (lateraler resp. paravaginaler Defekt)

- zusätzliche Genitalpathologie mit geplantem abdominalem Vorgehen

- Z.n. erfolgloser TVT-Einlage

- zu erwartende ausgeprägte Vernarbungen der vorderen Vaginalwand nach vorausgegangener Kolporrhaphia anterior

\section{Prinzip}

Fixation der endopelvinen Faszie im Bereich der mid- und proximalen Urethra am Blasenhals an das Cooper-Ligament, womit die anatomische Position des Blasenhalses durch die indirekte Elevation und Suspension der Blasenhalsregion resp. der endopelvinen Faszie wie in einer Hängematte in eine ventrokraniale Position kommt

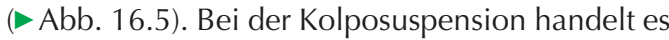
sich um den bisherigen Standard, wobei dazu viele Modifikationen zählen. Cowan und Morgan haben die Technik mittels lockerer Aufhängung mit freiverlaufenden Fäden zur Reduktion der Bla-

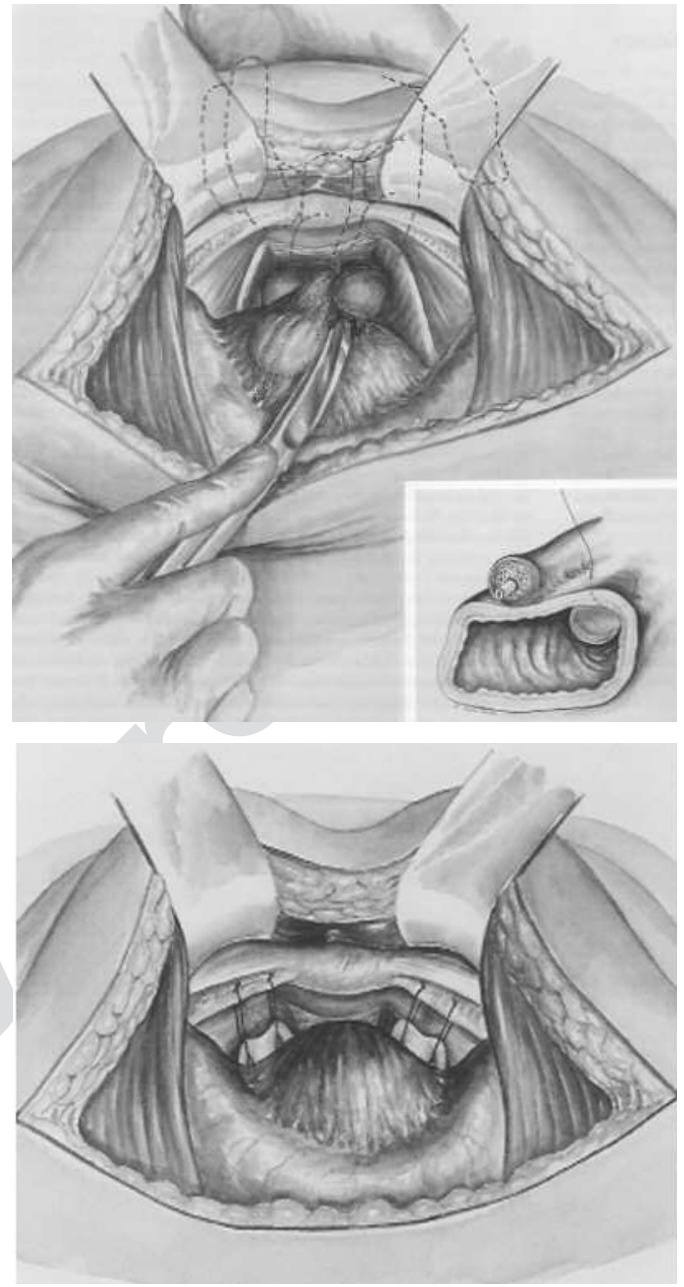

Abb. 16.5 Abdominale Kolposuspension nach Burch (aus Hirsch et al. 1999)

senentleerungsstörungen modifiziert. Kleinere Defekte der vorderen Vaginalwand werden beim Burch mitkorrigiert, und durch zusätzlich durch die parietalen Anteile des Arcus tendineus fasciae pelvis gelegte Fäden kann ein Lateraldefekt suffizient verschlossen werden. (Wird der Lateraldefekt auf diese Weise mit oder ohne Fixation der Fäden am Cooper-Ligament in Höhe des Blasenbodens [Level II nach DeLancey] fortgesetzt, sprechen wir von einer lateralen Rekonstruktion [paravaginal repair] zur Behebung der durch einen Lateraldefekt bedingten Zystozele.) Der Eingriff kann offen oder laparoskopisch erfolgen. Letzteres reduziert die Hospitalisations- und Rekonvaleszensdauer. We- 
sentliche Unterschiede im Outcome zwischen den beiden Vorgehensweisen finden sich nicht. Zur Suspension wird heute meist nichtabsorbierbares Fadenmaterial wie Ethibond oder GoreTex eingesetzt. Eine Überkorrektur des vesikourethralen Beta-Winkels ist zu vermeiden, und die Urethra soll so positioniert werden, dass man bequem zwei Finger zwischen dem Os pubis und der Urethra einführen kann. Therapieversager sind mitunter auf technische Fehler zurückzuführen, wie Setzen der Fäden in die Blase anstelle in die endopelvine Faszie, zu laterale Positionierung von der Urethra oder zu distal, exzessive Spannung resp. Elevation der Urethra und unzureichend tiefes Setzen der Nähte in die endopelvine Faszie mit folglich Ausreißen der Fäden. Zum Ausschluss intraoperativer Blasen- oder Harnröhrenverletzungen und in der Blase liegenden Fadenmaterials kann eine Kontrollzystoskopie durchgeführt. Bei Operationsende wird ein Katheter (oder eine suprapubische Ableitung für das postoperative Blasentraining) gelegt und für einige Tage belassen.

\section{Erfolg}

Ein systematischer Review zeigt für die offene Kolposuspension eine geringere Lang- und Kurzzeitversagerrate als bei vorderer Raffung und Nadelsuspensionen, mit Kontinenz von 85 bis $90 \%$ nach einem Jahr und $70 \%$ nach fünf Jahren. Es fanden sich keine signifikanten Unterschiede zwischen der Kolposuspension und den suburethralen Schlingen (Lapitan et al. 2005). Mittlerweile liegen die 5-Jahresdaten der großen MulticenterRCT vor (Ward et al. 2008): Die TVT- und die Burch-Gruppe wiesen vergleichbar gute Kontinenzraten von $81 \%$ resp. $90 \%$ auf. Während bei der Burch-Gruppe eine Zunahme an Entero- und Rektozelen festgestellt wurde, fanden sich drei späte Bandkomplikationen beim TVT. In einem systematischen Review von sieben RCT zwischen laparoskopischer Kolposuspension nach Burch und TVT fanden sich ebenfalls keine signifikanten Unterschiede bis auf eine kürzere Operations- und Hospitalisationsdauer zugunsten des TVT (Dean et al. 2006). Außerdem ergaben sich für die Kolposuspension nach Burch urodynamisch bestätigte Langzeiterfolgraten von 70-90\%,. Bei Mischinkontinenz sinkt die Rate auf etwa $60 \%$. Weitere Risikofaktoren für schlechteres Outcome sind Frauen unter 50 Jahre mit einer hypotonen Urethra (maximaler Urethralverschlussdruck $<20 \mathrm{~cm} \mathrm{H}_{2} \mathrm{O}$ ),
Adipositas, Asthma, Alter $>65$ Jahre sowie Östrogenmangel. Die Kolposuspension kann in der Rezidivsituation wiederholt werden, ist aber technisch aufgrund der retropubischen Fibrose aufwendiger. Die Kontinenzrate liegt bei $90 \%$ für den Ersteingriff und $82 \%$ für den wiederholten Eingriff (Dainer et al. 1998).

\section{Komplikationen}

Dazu zählen Harnverhalt, Detrusorüberaktivität in $17 \%$ (Vierhout et al. 1992), Blasen- oder Ureterenläsion, Infektion, Blutung und Rekto- resp. Enterozelen in $13 \%$, bedingt durch Schaffung eines Raumes im vaginalen Cul-de-sac durch Anheben der vorderen Vaginalwand zum oberen Schambeinast hin (Stanton et al. 1984). So ist bei Frauen, die nebst Kolposuspension nach Burch auch eine Hysterektomie erhalten, die apikale Aufhängung zu diskutieren. Blasenentleerungsstörungen und De-novo-Urge scheinen im Zusammenhang mit einer zu starken Elevation des Blasenhalses und Kompression der Urethra zu stehen. Leider kann dies intraoperativ klinisch zu wenig gut geprüft werden. Harnverhalt löst sich in der Regel innerhalb einer Woche (Katheterpflichtigkeit 5 bis 60 Tage, durchschnittlich 10 Tage [Korda et al. 1989]). Nur eine transfusionsbedürftige Blutung trat unter 3.500 Eingriffen auf (Dainer et al. 1999).

\subsubsection{Bulking agents}

\section{Indikation}

- Belastungsinkontinenz bei hypotoner, hypomobiler oder fixierter Urethra (intrinsische Sphinkterinsuffizienz)

- Rezidivinkontinenz resp. Zustand nach mehreren Inkontinenzeingriffen (voroperierte starre hypotone Urethra)

- multimorbide Patientin

\section{Prinzip}

Merke: Durch periurethrale Injektion eines Bulking agents kommt es zu einer lokalen Ausdehnung von periurethralem Gewebe, wodurch einerseits die Urethra eingeengt und andererseits die Drucktransmission in der proximalen oder mittleren Urethra erhöht wird ( Abb. 16.6). 


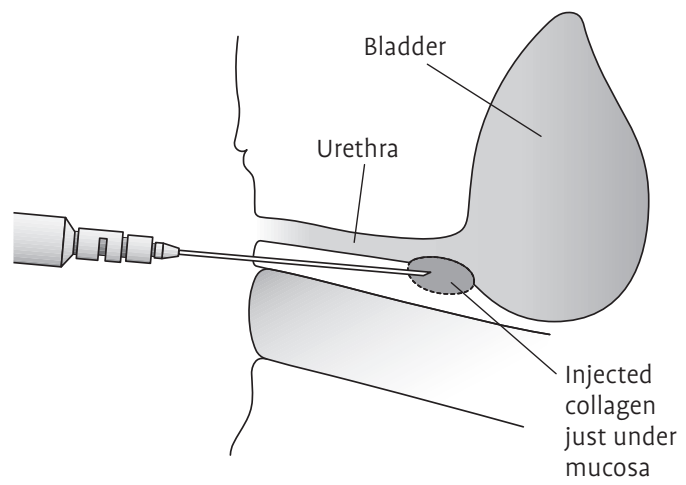

Abb. 16.6 Periurethrale Injektion eines Bulking agents in Blasenhalsnähe am Beispiel von Kollagen

Diskutiert wird eine durch die Injektion des Bulking agents erhöhte Kraft des Sphinkters, indem das Material als Füllvolumen die Länge der Muskelfibern erhöht (Klarskov et al. 2008). Es handelt sich hierbei um die am wenigsten invasive Inkontinenztechnik, die im ambulanten Setting und in Lokalanästhesie durchgeführt werden kann. Die Injektion kann unter urethroskopischer Sicht oder blind über eine Führungskanüle, periurethral neben die Urethra oder unter deren Submukosa erfolgen. Aktueller Gegenstand der Diskussion ist, ob Bulking agents am Blasenhals oder - in Analogie zu den modernen Schlingen - midurethral injiziert werden sollen (Kuhn et al. 2008). Nach einem Jahrhundert Erfahrung stehen mittlerweile verschiedene Produkte zur Injektion zur Verfügung. Standardprodukt, mit dem andere Bulking agents verglichen werden müssen, ist das Contigen $^{\mathrm{TM}}$, bestehend aus kreuzvernetzem bovinem Kollagen. Dieses ist biodegradierbar, weswegen der Bulking-Effekt vorübergehend ist und Reinjektionen erfordert. Nichtdegradierbare Produkte sind beispielsweise das Macroplastique aus Silikon. Verschiedene Bulking agents sind bereits vom Markt genommen worden (Teflon; Hyaluronsäure, Zuidex, Tegress). Ein aktuelles Bulking agent ist das Polymer Polyacrylamid Hydrogel (Bulkamid $\left.{ }^{\circledR}\right)$, es liegen jedoch nur kleine, nicht randomisierte Fallserien vor.

\section{Erfolgsraten}

Eine einzige prospektiv randomisierte Studie verglich die Kollageninjektion mit operativen Techniken (Corcos et al. 2005). Objektive Kontinenz, definiert als 24-Stunden-Padtest $\times 2,5$ g, konnte nach zwölf Monaten nur bei $53 \%$ in der Kollagengruppe, jedoch bei $72 \%$ der Frauen in der chirurgischen (Kolposuspension nach Burch, vaginale Nadelsuspensionen, Schlingenoperation) erzielt werden. Hingegen war die Lebensqualität in der Kollagengruppe statistisch nicht signifikant schlechter $(p=0,228)$ und die Komplikationsrate sogar signifikant geringer als in der chirurgischen $(p=0,03)$, was der Kollageninjektion zugute gehalten werden muss. Jedenfalls fehlen allen Methoden Langzeitresultate. Durch die unterschiedlichen Materialien der Bulking agents ist ein direkter Vergleich schwierig bis unmöglich, doch scheint kein Material hinsichtlich Effektivität überlegen zu sein (Lightner et al. 2001, Mayer et al. 2007). Die Ansprechraten schwanken zwischen $50 \%$ und $80 \%$ bei Nachbeobachtungen von ein bis zwei Jahre. Die jüngste Cochrane-Übersicht schließt damit, dass Bulking agents eine nützliche Option zur kurzfristigen Linderung der Symptome bei Frauen mit Komorbiditäten und Kontraindikation für eine Narkose darstellen (Keegan et al. 2007).

\section{Komplikationen}

Nebst postoperativem Harnverhalt (Schulz et al. 2004) stehen die materialabhängigen Komplikationen im Vordergrund. So ist das Kollagenprodukt Contigen $^{\mathrm{TM}}$ allergen, weswegen vor der Anwendung ein Hauttest durchgeführt werden muss. Höhere Komplikationsraten sind beim Einsatz von Bulking agents auf Karbonbasis wie das Durasphere $^{\circledR}$ beschrieben, bei dem das Material migrieren oder Granulome bilden kann. Silikone werden mit Autoimmunerkrankungen in Verbindung gebracht (Van Kerrebroeck et al. 2003). Vom Einsatz von autologem Fett und Polytetrafluoroethylen (Teflon) wird abgeraten (Keegan et al. 2007).

\subsubsection{Diskussion der Schlingentechnik: retropubischer oder transobtura- torischer Zugang?}

Das TVT zeichnet sich durch eine standardisierte Technik nach Kochbuch aus, die im Gegensatz zum bisherigen Goldstandard der Kolposuspension nach Burch in Lokalanästhesie durchführbar ist. Der kurzstationäre Aufenthalt trägt zur Reduktion der Kosten bei. Mittlerweile sind über $600 \mathrm{Pu}-$ blikationen zum TVT abrufbar, darunter mehrere 
Tab. 16.8 Gegenüberstellung der Komplikationen bei TVT und TO, basierend auf großen Registern. Quellen: Costa et al. 2004, De Leval et al. 2003 und 2004, Heidler et al. 2005, Kuuva et al. 2002, Tamussino et al. 2001 und 2007, Ward et al. 2002

\begin{tabular}{lll}
\hline Komplikationen & TVT & TO \\
\hline $\mathrm{n}$ & 9.693 & 8.365 \\
Blasenperforation & $3 \%$ & $0,5 \%$ \\
Urethraperforation & $0,02 \%$ & $0,3 \%$ \\
Blutung & $1,5 \%$ & $3,3 \%$ \\
Laparotomie & & \\
(intestinale Verletzungen) & $0,7 \%$ & $0 \%$ \\
Blasenentleerungsstörung & $4,6 \%$ & $15,9 \%$ \\
Harnretention & $1,7 \%$ & $2,8 \%$ \\
De-novo-OAB & $6,9 \%$ & $5,0 \%$ \\
Banderosion & $1 \%$ & $3,6-20 \%$ \\
\hline
\end{tabular}

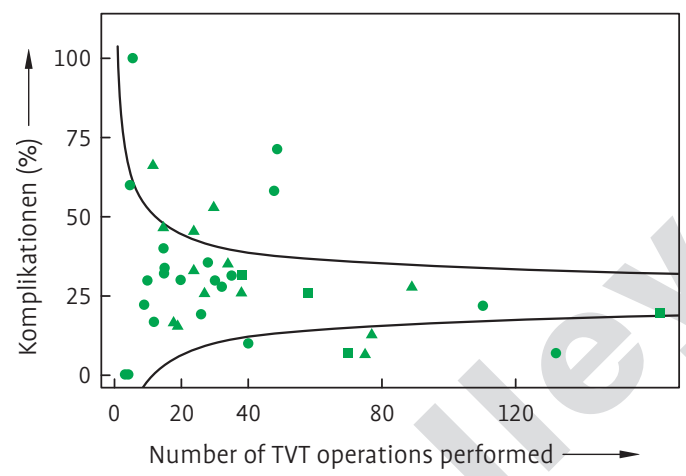

Abb. 16.7 Verhältnis Anzahl Komplikationen zu TVTOperationen in verschiedenen finnischen Spitälern: $\square=$ Universitätshospital, ? = Zentralhospital, • = örtliches Hospital (Kuuva und Nilsson 2002)

randomisiert kontrollierte Studien im Vergleich zum Standard Kolposuspension: Das TVT verursacht - bei gleicher Kontinenzrate - weniger Harnretention und Entero- resp. Rektozelen. Tatsächlich aber ergab sich mit zunehmender Verbreitung der Technik eine Zunahme von Komplikationen als Folge der Passage der Nadeln durch das kleine Becken, allen voran Blasenperforationen in etwa $3 \%\left({ }_{T} a b .16 .8\right)$. Allerdings variiert die Komplikationsrate von Studie zu Studie und hier wohl insbesondere von Operateur zu Operateur. Tatsächlich unterliegt die TVT-Einlage einer Lernkurve mit einer Mindesteinlagezahl von 40 Schlingenoperationen pro Jahr (Gold et al. 2007, Groutz et al. 2002, Lebret et al. 2001, McLennan et al. 2005). Eine Multicenterstudie zeigte eine Komplikations- rate von 40 \% für Zentren mit weniger als 15 TVTOperationen pro Jahr und eine Stabilisierung auf $14 \%$ bei Zentren mit über 15 Operationen (Kuuva et al. 2002) ( $\triangleright$ Abb. 16.7).

Zur Vermeidung der Blasenverletzung wurde der transobturatorische Zugang entwickelt, der ebenfalls wie das TVT eine midurethrale Stabilisierung bezweckt. Die postulierte bei gleich bleibender Kontinenzrate geringere Komplikationsrate an Blasenentleerungsstörungen und De-novo-Urge aufgrund der geringeren Obstruktion durch das transobturatorische Band konnte nur bedingt bestätigt werden, wogegen die Rate an Banderosionen, teils materialbedingt, teils aber durch den operativen Zugang bedingt, zunahm. Gerade bei Frauen mit hohen lateralen Sulci ist der transobturatorische Zugang deutlich erschwert und lässt eine höhere Banderosionsrate erwarten, wie unsere eigene Erfahrung zeigt $(\downarrow$ Tab. 16.9). Zudem ist die Machbarkeit einer operativen Revision zu berücksichtigen. Dies gilt besonders für Inkontinenzeingriffe, welche die Lebensqualität wiederherstellen und nicht eine bedrohliche Krankheit abwenden sollen. Ein weiteres Argument für den transobturatorischen Zugang ist die kürzere Operationsdauer, die allerdings mit dem Verzicht auf die Zystoskopie erklärt werden kann. So fragt sich, worin die Relevanz der um einige Minuten kürzeren Operationszeit besteht, während man gleichzeitig eine zusätzliche Sicherheit und Diagnostik durch die Zystoskopie erzielen kann. Da sich retropubische und transobturatorische Schlingen praktisch hinsichtlich der Kontinenz nicht unterscheiden, ist das Augenmerk auf die Komplikationen zu richten. Stehen beim TVT die perioperativen Komplikationen im Vordergrund, so sind es bei den transobturatorischen die mittel- oder langfristigen wie Banderosionen oder Geschlechtsverkehr assoziierte Beschwerden. Letztlich ist es der Operateur, der sich auf eine Technik festlegen muss und das Vorgehen mit der Patientin zu besprechen hat.

\subsubsection{Postoperative Kontrolle}

Das postoperative Follow-up $(\checkmark$ Tab. 16.10) richtet sich nach dem Eingriff resp. den postoperativen Komplikationen. Eine Kontrolle durch den Operateur selbst gibt ein direktes Feedback . In der Regel führen wir eine erste Wund- und Restharnkontrolle nach zwei bis vier Wochen durch. Gegebenenfalls muss eine liegende suprapubische Harnableitung 
Tab. 16.9 Vergleich ausgewählter Inkontinenzoperationen

\begin{tabular}{|c|c|c|c|c|c|c|}
\hline Zugang & Technik & Indikation & Erfolg & Komplikationen & Langzeit & Bemerkungen \\
\hline vaginal & TVT & $\begin{array}{l}\text { Belastungs- } \\
\text { inkontinenz } \\
\text { Rezidivsituation }\end{array}$ & $90 \% * *$ & $\begin{array}{l}\text { Blasenperforation 1-3\% } \\
\text { Blasenentleerungs- } \\
\text { störung } \\
\text { Darmverletzung } \\
\text { Hämorrhagie }\end{array}$ & De-novo-Urge & $\begin{array}{l}\text { Kurzhosp } 3 \text { Tage } \\
\text { Eingriff in LA/Anal- } \\
\text { gosedation intraop. } \\
\text { Kontrolle der } \\
\text { "Bandspannung" }\end{array}$ \\
\hline vaginal & TO & $\begin{array}{l}\text { Belastungs- } \\
\text { inkontinenz }\end{array}$ & $\begin{array}{l}80 \% \\
\text { (subj. } 92 \% \text { ) } \\
* * * *\end{array}$ & $\begin{array}{l}\text { Urethral- } \\
\text { perforation } 1 \% \\
\text { Blasenentleerungs- } \\
\text { störung }\end{array}$ & $\begin{array}{l}\text { Banderosion } 6 \% \\
\text { Dyspareunie/Ge- } \\
\text { schlechtsverkehr } \\
\text { assoziierte Be- } \\
\text { schwerden } 7 \%\end{array}$ & $\begin{array}{l}\text { Kurzhosp } 3 \text { Tage } \\
\text { Eingriff in LA/Anal- } \\
\text { gosedation intraop. } \\
\text { Kontrolle der } \\
\text { "Bandspannung" } \\
\text { bei TO noch keine } \\
\text { Langzeitresultate } \\
\text { vorliegend }\end{array}$ \\
\hline $\begin{array}{l}\text { abdo- } \\
\text { minal } \\
\text { laparo- } \\
\text { skopisch }\end{array}$ & $\begin{array}{l}\text { Burch/ } \\
\text { Cowan }\end{array}$ & $\begin{array}{l}\text { Belastungs- } \\
\text { inkontinenz } \\
\text { bei gleichzei- } \\
\text { tig bestehendem } \\
\text { lateralem Defekt } \\
\text { (Zystozele) }\end{array}$ & $\begin{array}{l}90 \% * * * \\
78 \% *\end{array}$ & $\begin{array}{l}\text { Blasenentleerungs- } \\
\text { störungen 15-20\% }\end{array}$ & $\begin{array}{l}\text { Rekto- und Ente- } \\
\text { rozelen in } 7-35 \% \\
\text { De-novo-Urge } \\
10-15 \%\end{array}$ & $\begin{array}{l}\text { abdominaler Eingriff } \\
\text { in Narkose keine } \\
\text { intraop. Erfolgskon- } \\
\text { trolle möglich Hosp. } \\
\text { 5-7 Tage Eingriff i. } \\
\text { Ggs. zu den Schlin- } \\
\text { gen "unter Sicht" }\end{array}$ \\
\hline $\begin{array}{l}\text { peri- } \\
\text { urethal } \\
\text { trans- } \\
\text { urethral }\end{array}$ & $\begin{array}{l}\text { Bulking } \\
\text { agents }\end{array}$ & $\begin{array}{l}\text { intrinsische } \\
\text { Sphinkterinsuffi- } \\
\text { zienz, hypo- } \\
\text { mobile Urethra } \\
\text { Rezidivsituation } \\
\text { schlechter Allge- } \\
\text { meinzustand }\end{array}$ & $26-75 \%$ & $\begin{array}{l}\text { Harnverhalt } \\
\text { (Schulz et al. 2004) }\end{array}$ & $\begin{array}{l}\text { Fremdkörper- } \\
\text { granulome, } \\
\text { Abszesse }\end{array}$ & $\begin{array}{l}\text { minimalinvasiver } \\
\text { Eingriff Alternative } \\
\text { zur zumindest kurz- } \\
\text { fristigen Symptom- } \\
\text { behebung bei Pa- } \\
\text { tientinnen mit Ko- } \\
\text { morbiditäten und } \\
\text { Risikofaktoren für } \\
\text { Eingriffe in Narkose. } \\
\text { Injektion muss ggf. } \\
\text { zwei- bis dreimal } \\
\text { wiederholt werden } \\
\text { (Keegan et al. 2007) }\end{array}$ \\
\hline
\end{tabular}

\footnotetext{
* 20 Jahre Follow-up

** 11 Jahre Follow-up (Nilsson et al. 2008)

*** 5 Jahre Follow-up (Ward et al. 2008)

**** 2 Jahre Follow-up (Giberti et al. 2007)
}

kontrolliert resp. gezogen werden. Die Patientin kann bei reizlosen Wundverhältnissen und $v$. a. bei restharnfreier Spontanmiktion aus der Nachkontrolle entlassen werden.

Nach Implantation alloplastischer Schlingen ist eine Verlaufskontrolle nach sechs bis zwölf Monaten wünschenswert. Spätestens wenn die Patientin Beschwerden wie Dyspareunie resp. Geschlechtsverkehr assoziierte Beschwerden, aber auch, wie neuerdings in der englischen Literatur beschrieben, Hispareunia, d. h. Schmerzen beim Partner (Brubaker et al. 2006), bei störendem vaginalen Ausfluss, rezidivierenden Harnweginfekten oder De-novoUrge angibt, ist eine Verlaufskontrolle indiziert. Der niedergelassene Gynäkologe spielt hier die Schlüs- selrolle. Anlässlich der regulären Jahreskontrolle soll auf subjektive Beschwerden wie Beschwerden oder Schmerzen im Hüftbereich resp. Adduktorenbereich, schmerzhafte Außenrotation im Hüftgelenk, Dyspareunie, Fluor vaginalis und Symptome der überaktiven Blase geachtet werden. Bei Beschwerden ist eine Urinuntersuchung sowie eine Restharnkontrolle indiziert. Mittels Perinealsonographie kann bei Zustand nach Schlingenoperation gleich die suburethrale Bandlage evaluiert werden. Hinweise auf eine falsche oder obstruktive Bandlage sind blasenhalsnahe Position, ein Bandabstand $<3 \mathrm{~mm}$ zur Urethra (Kociszewski et al. 2008), ein Kinking (Abknicken) der Urethra durch das Band sowie ein Cshape des Bandes. Wichtig ist nebst der Spekulaun- 


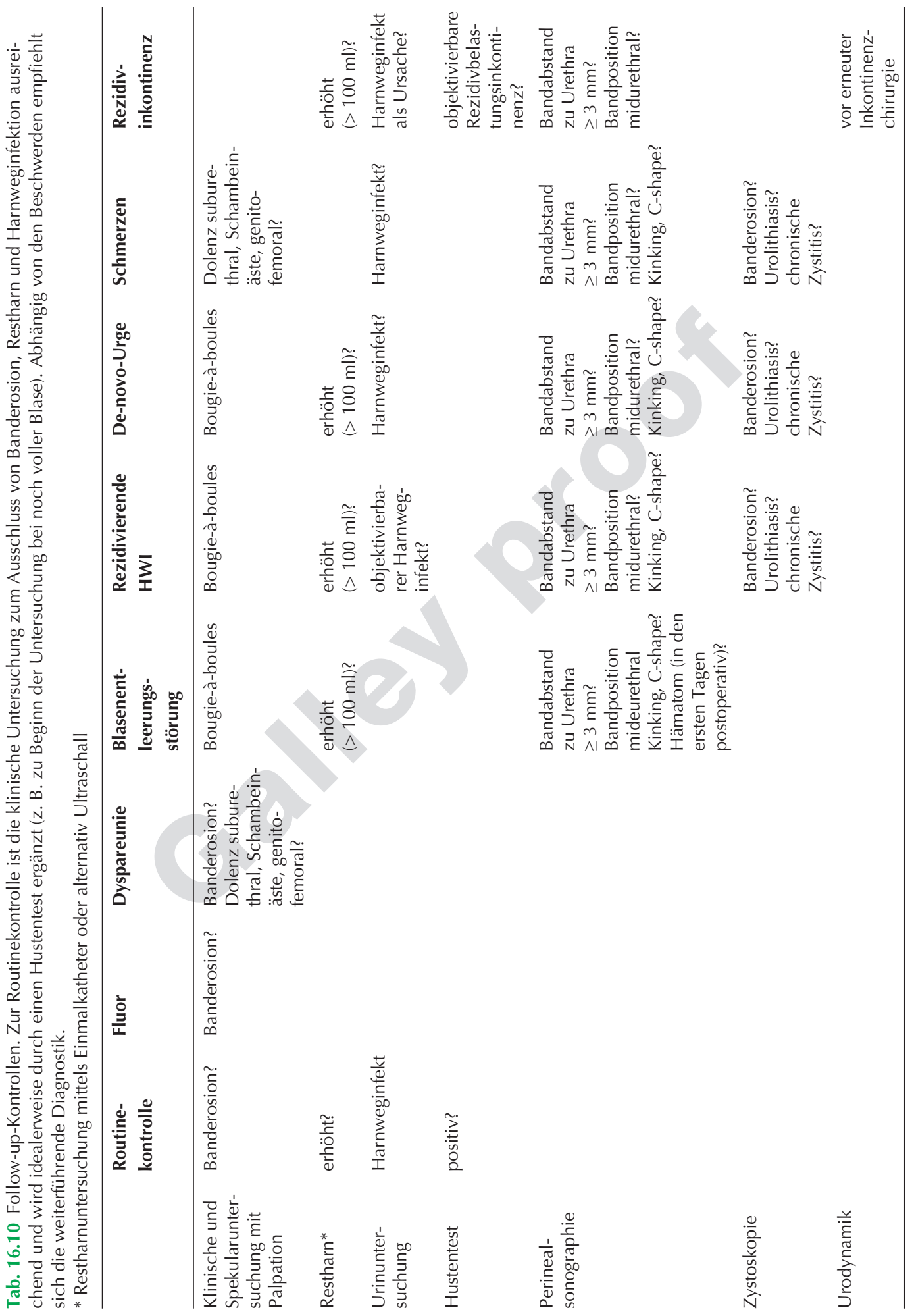


tersuchung v. a. die digitale Palpation, welche frühzeitig die Erosion ertasten lässt: Die feinen, durch die Vagina spießenden Mesh-Schlaufenenden der Schlingen sind häufig in den Rugae der Vagina nicht sichtbar, gerade wenn sie nicht dunkel gefärbt sind oder wenn die Übersicht erschwert ist (enger Introitus, Adipositas etc.). Bei rezidivierenden Harnweginfekten sollte eine Urethrozystoskopie zum Ausschluss einer Banderosion in die Blase oder Urethra durchgeführt werden. Kommt es zur Rezidivinkontinenz, ist eine erweiterte urogynäkologische Diagnostik inkl. Urodynamik (Füllungszystotonometrie, Urethrotonometrie, Uroflow, Urethrozystoskopie und Perinealsonographie) indiziert, um die Inkontinenz von einer Überlaufblase, Blasenfistel mit ständigem, belastungsunabhängigem Urinabgang, primärem Therapieversager (innerhalb der ersten drei Monate) oder einer echten Rezidivbelastungsinkontinenz zu unterscheiden.

\section{Literatur}

Abrams P, Cardozo L, Fall M, Griffiths D, Rosier P, Ulmsten U, van Kerrebroeck P, Victor A, Wein A, The standardisation of terminology of lower urinary tract function: report from the Standardisation Subcommittee of the International Continence Society. Am J Obstet Gynecol 2002;187(1): 116-26.

Aldridge $\mathrm{AH}$, Transplantation of fascia for the relief of urinary incontinence. Am J Obstet Gynecol 1942;44: 398-411.

Amid PK, Classification of biomaterials and their related complications in abdominal wall hernia surgery. Hernia 1997;1: 15-21.

Andersen JT, Blaivas J, Cardozo L, Thüroff J, Lower urinary tract rehabilitation techniques: Seventh report on the standardization of terminology of lower urinary tract function Neurourol Urodynam 992;11: 593-603.

Anger JT, Litwin MS, Wang Q, Pashos CL, Rodríguez LV, The effect of concomitant prolapse repair on sling outcomes. J Urol 2008;180(3): 1003-6.

AWMF, Leitlinien der Deutschen Gesellschaft für Geriatrie: Harninkontinenz. 4.10.2007. Leitlinienregister Nr.084/001.

AWMF, Leitlinien der Deutschen Gesellschaft für Gynäkologie und Geburtshilfe (DGGG), Arbeitsgemeinschaft Urogynäkologie und Beckenbodenrekonstruktion in der DGGG: Belastungsinkontinenz der Frau. Leitlinienregister Nr. 015/005

Balmforth J, Robinson D, Pelvic organ prolapse. In: Bø K, Berghmans B, Mørkved S, v. Kampen S, editors. Evidence-based physical therapy for the pelvic floor bridging science and clinical practice. Edinburgh: Churchill Livingstone Elsevier; 2007. p. 233-40.
Bergman A, Elia G, Three surgical procedures for genuine stress incontinence: five-year follow-up of a prospective randomized study. Am J Obstet Gynecol 1995;173(1): 66-71.

Borstad E, Abdelnoor M, Mogimi K, Sandved M, Majida M, Western K, Rokkones E, Paulsen AG, Staff AC, Kulseng-Hanssen S, Surgery for concomitant pelvic organ prolapse and urinary stress incontinence. A multicenter prospective randomized trial to compare the results of an incontinence procedure performed at the time of prolapse repair or 3 months after. Neurourol Urodyn 2008;27(7): 713.

Brubaker L, Cundiff GW, Fine P, Nygaard I, Richter HE, Visco AG, Zyczynski H, Brown MB, Weber AM; Pelvic Floor Disorders Network, Abdominal sacrocolpopexy with Burch colposuspension to reduce urinary stress incontinence. N Engl J Med 2006;354(15):1557-66.

Brubaker L, Nygaard I, Richter HE, Visco A, Weber AM, Cundiff GW, Fine P, Ghetti C, Brown MB, Two-year outcomes after sacrocolpopexy with and without burch to prevent stress urinary incontinence. Obstetrics and gynecology 2008;112(1):49-55.

Burch JC, Urethrovaginal fixation to Cooper's ligament for correction of stress incontinence, cystocele, and prolapse. Am J Obstet Gynecol 1961;81: 281-90.

Cardozo L, Staskin D, Textbook of Female Urology and Urogynaecology.Oxford: ISIS Medical Media; 2001.

Chaikin DC, Groutz A, Blaivas JG, Predicting the need for anti-incontinence surgery in continent women undergoing repair of severe urogenital prolapse. J Urol 2000;163(2): 531-4.

Cody J, Wyness L, Wallace S, Glazener C, Kilonzo M, Stearns S, McCormack K, Vale L, Grant A, Systematic review of the clinical effectiveness and cost-effectiveness of tension-free vaginal tape for treatment of urinary stress incontinence. Health technology assessment 2003;7(21): iii, 1-189.

Comiter CV, Surgery insight: management of failed sling surgery for female stress urinary incontinence. Nat Clin Pract Urol 2006;3(12): 666-74.

Costa P, Grise P, Droupy S, Monneins F, Assenmacher C, Ballanger P, Hermieu JF, Delmas V, Boccon-Gibod L, Ortuno C, Surgical treatment of female stress urinary incontinence with a trans-obturator-tape (T.O.T.) Uratape: short term results of a prospective multicentric study. Eur Urol 2004;46(1): 102-6; discussion 106-7.

Cowan W, Morgan HR, A simplified retropubic urethropexy in the treatment of primary and recurrent urinary stress incontinence in the female. Am J Obstet Gynecol 1979;133(3): 295-8.

de Leval J, Letters to the editor. Europ Urol 2004;46: 133-7.

de Leval J, Novel surgical technique for the treatment of female stress urinary incontinence: transobturator 
vaginal tape inside-out. Eur Urol 2003;44(6): 724-30.

Dean N, Herbison P, Ellis G, Wilson D, Laparoscopic colposuspension and tension-free vaginal tape: a systematic review. BJOG 2006;113(12): 1345-53.

Dean NM, Ellis G, Wilson PD, Herbison GP, Laparoscopic colposuspension for urinary incontinence in women. Cochrane database of systematic reviews 2006; 3: CD002239.

DeLancey JO. Structural support of the urethra as it relates to stress urinary incontinence: the hammock hypothesis. Am J Obstet Gynecol 1994;170(6): 1713-20.

Delorme E, Transobturator urethral suspension: miniinvasive procedure in the treatment of stress urinary incontinence in women). Prog Urol 2001;11(6): 1306-13.

Duckett JR, Vella M, Kavalakuntla G, Basu M, Tolerability and efficacy of duloxetine in a nontrial situation. BJOG 2007; 114(5):543-7.

Eberhardt J, Geissbühler V, Pessarbehandlung in der Urogynäkologie und Geburtshilfe. Gynäkol Prax 2002;26: 119-33.

Eliasson K, Edner A, Mattsson E, Urinary incontinence in very young and mostly nulliparous women with a history of regular organised high-impact trampoline training: occurrence and risk factors. Int Urogynecol J Pelvic Floor Dysfunct 2008;19(5): 687-96.

Enhörning G, Simultaneous recording of intravesical and intraurethral pressure: A study on urethral closure in normal and stress incontinent women. Acta Chir Scand 1961;Suppl 1: 276.

Fatton B, Is there any evidence to advocate SUI prevention in continent women undergoing prolapse repair? An overview. Int Urogynecol J Pelvic Floor Dysfunct 2009;20(2): 235-45.

Fonda D, Resnick NM, Colling J, Burgio K, Ouslander JG, Norton C, Ekelund P, Versi E, Mattiasson A, Outcome measures for research of lower urinary tract dysfunction in frail older people. Neurourol Urodyn 1998;17(3): 273-81.

Frangenheim P, Zur operativen Behandlung der Harninkontinenz der männlichen Harnröhre. Verh Dsch Ges Chirurgie, 43rd Congress 1914; 149.

Giberti C, Gallo F, Cortese P, Schenone M., Transobturator tape for treatment of female stress urinary incontinence: objective and subjective results after a mean follow-up of two years. Urology 2007;69(4): 703-7.

Glazener CM, Cooper K. Anterior vaginal repair for urinary incontinence in women. Cochrane database of systematic reviews 2001;1: CD001755.

Goebell R, Zur operativen Beseitigung der angeborenen Incontinentia Versicae (German). Dtschr Gynakol Urol 1910;2: 187-91.

Grady D, Brown JS, Vittinghoff E, Applegate W, Varner E, Snyder T, Postmenopausal hormones and inconti- nence: the Heart and Estrogen/Progestin Replacement Study. Obstet Gynecol 2001;97: 116-20.

Hay-Smith EJ, Dumoulin C. Pelvic floor muscle training versus no treatment, or inactive control treatments, for urinary incontinence in women. Cochrane Database Syst Rev 2006;(1): CD005654.

Hendrix SL, Cochrane BC, Nygaard IE, Handa VL, Barnabei VM, Igesia C, et al., Effects of estrogens with and without progestin on urinary incontinence. JAMA 2005;293: 935-48.

Hilton $\mathrm{P}$, Stanton SL, Urethral pressure measurement by microtransducer: the results in symptom-free women and those with genuine stress incontinence. $\mathrm{Br}$ J Obstet Gynaecol 1983;90: 919-33.

Hirsch HA, Käser O, Iklé FA unter Mitwirkung von Eva Neeser, Atlas der gynäkologischen Operationen. 6. unveränderte Auflage. Stuttgart: Georg Thieme Verlag; 1999.

Keegan PE, Atiemo K, Cody J, McClinton S, Pickard R, Periurethral injection therapy for urinary incontinence in women. Cochrane database of systematic reviews 2007;3(2): CD003881.

Kelly HA. Incontinence of urine in women. Urol Cutan Rev 1913;17: 291.

Kilonzo M, Vale L, Stearns SC, Grant A, Cody J, Glazener CM, Wallace S, McCormack K, Cost effectiveness of tension-free vaginal tape for the surgical management of female stress incontinence. Health Technol Assess 2004;20(4): 455-63.

Kölle D, Tamussino K, Hanzal E, et al. Bleeding complications with the tension-free vaginal tape operation. Am J Obstet Gynecol 2005;193: 2045-9.

Kuhn A, Stadlmayr W, Lengsfeld D, Mueller MD, Where should bulking agents for female urodynamic stress incontinence be injected?. Int Urogynecol J Pelvic Floor Dysfunct 2008;19(6): 817-21.

Kuuva N, Nilsson CG, A nationwide analysis of complications associated with the tension-free vaginal tape (TVT) procedure. Acta Obstet Gynecol Scand 2002;81(1): 72-7.

Lapitan MC, Cody JD, Grant A, Open retropubic colposuspension for urinary incontinence in women. Cochrane database of systematic reviews 2005;3: CD002912.

Laycock J, Clinical evaluation of the pelvic floor. In: Schüssler B, Laycock J, Norton PA, Stanton SL, editors. Pelvic floor re-education: principles and practice. London: Springer Verlag; 2002. p. 42-8.

Latthe PM, Foon R, Toozs-Hobson P, Transobturator and retropubic tape procedures in stress urinary in continence: a systematic review and meta-analysis of effectiveness and complications. BJOG 2007;114(5): 522-31.

Liapis A, Bakas P, Lazaris D, Creatsas G, Tension-free vaginal tape in the management of recurrent stress incontinence. Arch Gynecol Obstet 2004;269(3): 205-7. 
Liapis A, Bakas P, Salamalekis E, Botsis D, Creatsas G, Tension-free vaginal tape (TVT) in women with low urethral closure pressure. Eur J Obstet Gynecol Reprod Biol 2004;116(1): 67-70.

Lovatsis D, Gupta C, Dean E, Lee F, Tension-free vaginal tape procedure is an ideal treatment for obese patients. Am J Obstet Gynecol 2003;189(6): 1601-4; discussion 1604-5.

Marshall VF, Marchetti AA, Krantz KE, The correction of stress incontinence by simple vesicourethral suspension. Surg Gynecol Obstet 1949;88: 509-18.

Martin JL, Williams KS, Abrams KR, Turner DA, Sutton AJ, Chapple C, Assassa RP, Shaw C, Cheater F, Systematic review and evaluation of methods of assessing urinary incontinence. Health Technol Assess 2006;10(6): 1-132, iii-iv.

Martius H, Sphincter und Harnröhrenplastic aus dem Musculus Bulbocavernosus. Chirurgie 1929;1: 769.

Mattiasson A, Djurhuus JC, Fonda D, Lose G, Nordling J, Stöhrer M, Standardization of outcome studies in patients with lower urinary tract dysfunction: A report on general principles from the standardisation committee of the international continence society. Neurourol Urodynam 1998;17: 249-53.

McGuire EJ, Lytton B, Pubovaginal sling for stress incontinence. J Urol 1978;119: 82-4.

Meschia M, Barbacini P, Ambrogi V, Pifarotti P, Ricci L, Spreafico L, TVT-secur: a minimally invasive procedure for the treatment of primary stress urinary incontinence. One year data from a multi-centre prospective trial. Int Urogynecol J Pelvic Floor Dysfunct 2009;20(3): 313-7.

Meschia M, Pifarotti P, Spennacchio M, Buonaguidi A, Gattei $U$, Somigliana E, A randomized comparison of tension-free vaginal tape and endopelvic fascia plication in women with genital prolapse and occult stress urinary incontinence. Am J Obstet Gynecol 2004;190(3): 609-13.

Messelink B, Benson JT, Berghmans B, Bo, Corcos J, Fowler C, Laycock J, Lim PHC, van Lunsen R, Nijeholt GL, Pemberton J, Wang A, Van Kerrebroeck P, Standardization of terminology of pelvic floor muscle function and dysfunction: report from the Pelvic Floor Clinical Assessment Group of the International Continence Society. Neurourol Urodynam 2005;24: 374-80.

Miller JJ, Botros SM, Akl MN, Aschkenazi SO, Beaumont JL, Goldberg RP, Sand PK, Is transobturator tape as effective as tension-free vaginal tape in patients with borderline maximum urethral closure pressure? Am J Obstet Gynecol 2006;195(6): 1799-804.

Monga A, Petri E, Rizk DE, Sand PK, Schaer GN, Standardization and Terminology Committees IUGA/ICS, Joint IUGA/ICS Urogynecology Working Group, An International Urogynecological Association (IUGA) - International Continence Society (ICS) Joint report on the terminology for female pelvic floor dysfunc- tion. Int Urogynecol J, Neurourol Urodynam 2009; submitted.

Neuman M, Perioperative complications and early follow-up with 100 TVT-SECUR procedures. Journal of minimally invasive gynecology 2008;15(4): 480-4.

Nilsson CG, Palva K, Rezapour M, Falconer C, Eleven years prospective follow-up of the tension-free vaginal tape procedure for treatment of stress urinary incontinence. Int Urogynecol J Pelvic Floor Dysfunct 2008;19(8): 1043-7.

Pereyra AJ, A simplified surgical procedure for the correction of stress incontinence in women. Western journal of surgery, obstetrics, and gynecology 1959;67(4): 223-6.

Perucchini D, DeLancey JO, Ashton-Miller JA, Peschers $U$, Kataria T, Age effects on urethral striated muscle. I. Changes in numbers and diameter of striated muscle fibres in the ventral urethra. Am J Obstet Gynecol 2002;186: 351-5.

Petri E. Gynäkologische Urologie. 3. überarbeitete Auflage. Stuttgart: Thieme-Verlag; 2001.

Petros PE, Ulmsten UI. An integral theory of female urinary incontinence. Experimental and clinical considerations. Acta Obstet Gynecol Scand 1990;153 (suppl): 7-31.

Raz S, Modified bladder neck suspension for female stress incontinence. Urology 1981;17(1): 82-5.

Rezapour M, Falconer C, Ulmsten U, Tension-Free vaginal tape (TVT) in stress incontinent women with intrinsic sphincter deficiency (ISD) - a long-term follow-up. Int Urogynecol J Pelvic Floor Dysfunct 2001;12 (suppl 2): S12-14.

Rezapour M, UImsten UI. Tension-Free vaginal tape (TVT) in women with recurrent stress urinary incontinence - a long-term follow up. Int Urogynecol J Pelvic Floor Dysfunct 2001;12(suppl 2): S9-11.

Richter HE, Burgio KL, Goode PS, Borello-France D, Bradley CS, Brubaker L, Handa VL, Fine PM, Visco AG, Zyczynski HM, Wei JT, Weber AM; Pelvic Foor Desorders Network. Non-surgical management of stress urinary incontinence: ambulatory treatments for leakage associated with stress (ATLAS) trial. Clin Trials 2007;4(1): 92-101.

Rogers RG, Urinary stress incontinence in women. N Engl J Med 2008;358: 1029-36.

Sampselle CM, Brink CA, Wells TJ, Digital measurement of pelvic muscle strength in childbearing women. Nurs Res 1989;38: 134-8.

Schuessler B, Laycock J, Norton P, Stanton S, Pelvic floor re-education. London: Springer Verlag; 1994.

Schulz JA, Nager CW, Stanton SL, Baessler K, Bulking agents for stress urinary incontinence: short-term results and complications in a randomized comparison of periurethral and transurethral injections. Int Urogynecol J Pelvic Floor Dysfunct 2004;15(4): 261-5.

Shaikh S, Ong EK, Glavind K, Cook J, N'Dow JM, Mechanical devices for urinary incontinence in 
women. Cochrane Database Syst Rev 2006;3: CD001756.

Skinner MH, Kuan HY, Skerjanec A, Seger ME, Heathman M, O'Brien L, Reddy S, Knadler MP, Effect of age on the pharmacokinetics of duloxetine in women. Br J Clin Pharmacol 2004;57(1): 54-61.

Stamey TA, Endoscopic suspension of the vesical neck for urinary incontinence. Surgery, gynecology \& obstetrics 1973;136(4): 547-54.

Stoeckel W. Über die Verwendung der Musculi Pyramidales bei der operativen Behandlung der Incontinentia urinae. Zbl Gynäk 1917;41: 11.

Subak LL, Wing E, Smith West D, et al. Weight loss to treat urinary incontinence in overweight and obese women. N Engl J Med 2009;360: 481-90.

Sung VW, Schleinitz MD, Rardin CR, Ward RM, Myers $\mathrm{DL}$, Comparison of retropubic vs. transobturator approach to midurethral slings: a systematic review and meta-analysis. Am J Obstet Gynecol 2007;197(1): 3-11.

Sweeney DD, Chancellor MB, Treatment of stress urinary incontinence with duloxetine hydrochloride. Rev Urol 2005;7(2): 81-6.

Tamussino K, Hanzal E, Kölle D et al., Transobturator tapes for stress urinarty incontinence: Results of the
Austrian Registry. Am J Obstet Gynecol 2007;197: 634.e1-5.

Tamussino K, Hanzal E, Kölle D, Ralph G, Riss PA, The tension-free vaginal tape operation: Results of the Austrian registry. Obstet Gynecol 2001;98: 732-6.

Tanzberger R, Kuhn A, Möbs G, Der Beckenboden Funktion, Anpassung und Therapie. Das TanzbergerKonzept. München: Urban \& Fischer in Elsevier; 2004.

Ulmsten U, Petros P, Intravaginal slingplasty (IVS): an ambulatory surgical procedure for treatment of female urinary incontinence. Scand J Urol Nephrol 1995;29(1): 75-82.

van Kerrebroeck P, ter Meulen F, Farrelly E, Larsson G, Edwall L, Fianu-Jonasson A, Treatment of stress urinary incontinence: recent developments in the role of urethral injection. Urol Res 2003;30(6): 356-62.

Ward KL, Hilton P; UK and Ireland TVT Trial Group, Tension-free vaginal tape versus colposuspension for primary urodynamic stress incontinence: 5 -year follow up. BJOG 2008;115(2): 226-33.

Zivkovic F, Tamussino K. Pieber D, Haas J, Body mass index and outcome of incontinence surgery. Obstet Gynecol 1999;93: 753-6. 